Ende der 1980er Jahre erreichte die "Gorbimanie“ in der Bundesrepublik Deutschland ihren Höhepunkt. Der Generalsekretär des Zentralkomitees der Kommunistischen Partei der Sowjetunion galt als Hoffnungsträger für ein Ende des Kalten Kriegs und eine Überwindung der Teilung Europas. Hermann Wentker zeigt auf, warum gerade die Grünen Michail Gorbatschow zurückhaltender begegneten als andere politische Kräfte in der alten Bundesrepublik, er erklärt, weshalb sie in ihrer Einschätzung zwischen Zustimmung und Kritik schwankten, und er fragt danach, welche Rolle die Auseinandersetzungen zwischen den „Realos“ und den „Fundis“ unter den Grünen dabei spielten.

Hermann Wentker

\title{
Die Grünen und Gorbatschow
}

Metamorphosen einer komplexen Beziehung 1985 bis 1990

\section{Einleitung}

Als der sowjetische Staats- und Parteichef Michail Gorbatschow vom 13. bis zum 15. Juni 1989 zu einem Staatsbesuch in der Bundesrepublik weilte, empfingen ihn die Grünen mit einem offenen Brief. Das in der Frankfurter Rundschau abgedruckte Schreiben enthielt eine differenzierte Auseinandersetzung mit Gorbatschows Politik aus „grüner“ Perspektive. Die Verfasser begrüßten seine Abrüstungspolitik uneingeschränkt und unterstützten die „Politik der Anerkennung des territorialen Status quo in Europa“ - einschließlich der deutschen Teilung unter „Aufgabe von politischen und wirtschaftlichen Hegemonieansprüchen in der BRD“. Kritik übten die Grünen an einzelnen autoritären Erscheinungen und dem gewaltsamen Umgang mit Protesten im Kaukasus, wenngleich sie „die Ausbrüche von Nationalismus" mit Sorge betrachteten. Grundsätzlich begrüßten sie die Einleitung von Wirtschaftsreformen, wollten allerdings „von Anfang an ökologische und soziale Kriterien ins Zentrum rücken“. Daher standen sie einigen westdeutsch-sowjetischen Wirtschaftsverträgen skeptisch gegenüber, „weil in ihnen kein neues Denken im Verhältnis Ökologie-Ökonomie zu entdecken“ sei ${ }^{1}$. Die Grünen setzten sich damit von der allgemeinen „Gorbimanie“ dieser Tage ebenso ab wie von der Deutschlandpolitik der Bundesregierung, die am Wiedervereinigungsgebot des Grundgesetzes festhielt.

Um diese zwischen Sympathie und Kritik oszillierende Position der Grünen zu verstehen, genügt es nicht, das nähere Umfeld des Staatsbesuchs vom Juni 1989 auszuleuchten. Es gilt vielmehr, das Verhältnis der Grünen zur Sowjetunion in den Jahren 1985 bis 1990 insgesamt zu betrachten. Dies soll in diesem Beitrag sowohl unter einer perzeptionsgeschichtlichen als auch unter einer transnatio-

1 „Herr Gorbatschow, wie soll das gemeinsame Haus denn aussehen?“, in: Frankfurter Rundschau vom 14.6.1989. 
nalen Fragestellung geschehen. Am Anfang steht die Frage, welches Bild sich die Grünen von Gorbatschow machten, wobei festzuhalten ist, dass der sowjetische Spitzenpolitiker ein Mann mit vielen Gesichtern war, der sich zwischen seinem Amtsantritt als Generalsekretär der Kommunistischen Partei der Sowjetunion (KPdSU) im März 1985 und den Umwälzungen des Jahres 1990 erheblich wandelte. Es fiel westlichen Beobachtern daher generell nicht leicht, ihn richtig einzuschätzen. Was die Grünen angeht, so wurde die Meinungsbildung durch ihre verwirrende Vielstimmigkeit und ihr besonderes Selbstverständnis zusätzlich erschwert: Angetreten als „Antiparteien-Partei“, avancierten sie ab Mitte der 1980er Jahre zunehmend zu einer „ökologischen Reformpartei“2, die sich außerdem als politisch-parlamentarischer Arm der Anti-Atomkraft- und der Friedensbewegung betrachtete ${ }^{3}$. Dieser Prozess war von heftigen innerparteilichen Auseinandersetzungen insbesondere zwischen zwei Gruppierungen geprägt. Auf der einen Seite standen die sogenannten Realpolitiker oder „Realos“, die zur Umsetzung ihrer Ziele eine Koalition mit der SPD und eine Regierungsbeteiligung nicht ausschlossen, um auf diese Weise das politische System der Bundesrepublik zu verändern. Auf der anderen Seite sprachen sich die Fundamentalisten oder „Fundis“ gegen diesen Kurs aus, da sie den Status quo vor allem mit Hilfe der sozialen Bewegungen überwinden wollten; das Parlament diente ihnen lediglich als Bühne, um ihren Anliegen zusätzlich Gehör zu verschaffen. Der transnationale Aspekt des vorliegenden Aufsatzes ergibt sich vor allem daraus, dass es sich bei den Grünen um nichtstaatliche Akteure handelte, die überdies nicht nur Beziehungen zur sowjetischen Führung unterhielten, sondern auch an Kontakten zu Dissidenten und den immer wichtigeren „informellen Gruppen“4 interessiert waren. Aus dieser Parallelität resultierten Spannungen, die die transnationalen Beziehungen der Grünen in die Sowjetunion zu einem besonders reizvollen Untersuchungsobjekt machen.

Nachdem sich zu Beginn der 1990er Jahre vor allem die Politikwissenschaft mit den Grünen als Phänomen zwischen sozialer Bewegung und politischer Partei befasst hat ${ }^{5}$, sind sie seit einiger Zeit auch Gegenstand der historischen Forschung. Eine zentrale Quelle für die Anfangsjahre bildet die Edition der Protokolle der

\footnotetext{
${ }^{2}$ Vgl. Silke Mende, Von der „Anti-Parteien-Partei“ zur „ökologischen Reformpartei“. Die Grünen und der Wandel des Politischen, in: Meik Woyke (Hrsg.), Wandel des Politischen. Die Bundesrepublik während der 1980er Jahre, Bonn 2013, S. 277-319.

${ }^{3}$ Vgl. Saskia Richter, Der Protest gegen den NATO-Doppelbeschluss und die Konsolidierung der Partei Die Grünen zwischen 1979 und 1983, in: Philipp Gassert/Tim Geiger/Hermann Wentker (Hrsg.), Zweiter Kalter Krieg und Friedensbewegung. Der NATO-Doppelbeschluss in deutsch-deutscher und internationaler Perspektive, München 2011, S. 229-245, hier S. 243245.

${ }^{4}$ Bei den „informellen Gruppen“ handelte es sich um gesellschaftliche Initiativen, die eine eigene, nicht notwendigerweise dissidentische Subkultur in der Sowjetunion bildeten. Unter Gorbatschow kam es zu einer starken Ausweitung dieser Szene; vgl. Yuliya von Saal, KSZE-Prozess und Perestroika in der Sowjetunion. Demokratisierung, Werteumbruch und Auflösung 1985-1991, München 2013, S. 86-100.

${ }^{5}$ Vgl. Joachim Raschke, Die Grünen. Wie sie wurden, was sie sind, Köln 1993; Rudolf van Hüllen, Ideologie und Machtkampf bei den Grünen, Bonn 1990; Hubert Kleinert, Aufstieg und
} 
Fraktion der Grünen im Bundestag zwischen 1983 bis 19876; grundlegend ist auch Josef Boyers Handbuchartikel über die Mitgliedschaft und Sozialstruktur der alternativen Partei ${ }^{7}$. Eine wichtige Untersuchung zur Geschichte der Gründungsgrünen hat kürzlich Silke Mende vorgelegt ${ }^{8}$; dabei spielte Petra Kelly eine herausragende Rolle, die inzwischen auch durch eine wissenschaftliche Biographie gewürdigt worden ist ${ }^{9}$. Was die Vorstellungen und Aktivitäten der Grünen auf verschiedenen Politikfeldern betrifft, so hat sich Mende neben den für die Grünen besonders relevanten Themen Wachstum und Fortschritt sowie Integration und „multikulturelle Gesellschaft“ auch dem Thema Sozialstaatlichkeit im Wandel gewidmet ${ }^{10}$. Daneben ist vor kurzem die Deutschlandpolitik der Grünen in den 1980er Jahren eingehend untersucht worden ${ }^{11}$. Etwas älter ist die politikwissenschaftliche, auch auf Archivmaterial beruhende Studie des ehemaligen Grünen-Politikers Ludger Volmer über das Verhältnis seiner Partei zur Außenpolitik. Die Grünen orientierten sich Volmer zufolge in den 1980er Jahren vor allem nach Westen; Osteuropa erschien den meisten von ihnen „als opak und undurchschaubar“. Deutlich wird, dass sich die Grünen in einem Dilemma befanden: Sie sahen im „real existierenden Sozialismus“ zwar keine Alternative zu den Verhältnissen in der Bundesrepublik, wollten aber weder als Verteidiger des von ihnen heftig kritisierten westdeutschen Systems noch als Antikommunisten erscheinen ${ }^{12}$.

Hintergrund dieses Dilemmas war der „Angst-Diskurs“ der Friedensbewegung zu Beginn der 1980er Jahre. Die durchaus reale, aber zugleich kultivierte Furcht vor einem Atomkrieg richtete sich dabei auch gegen diejenigen, die ein nukleares Inferno auslösen konnten. Dabei handelte es sich de facto um eine „halbierte Angst“, die sich sehr viel stärker gegen die Strategie der NATO und deren vermeintliche Kriegsvorbereitungen als gegen die Sowjetunion richtete. Denn Angst vor „den Russen“ war eine „falsche Angst“; das Feindbild Sowjetunion, das auch von anderen Parteien gezeichnet wurde, sahen Friedensbewegung und Grüne mehrheitlich als schädlich an, da es die westliche Aufrüstung rechtfertigte und

Fall der Grünen. Analyse einer alternativen Partei, Bonn 1992; Markus Klein/Jürgen Falter, Der lange Weg der Grünen, München 2003.

${ }^{6}$ Vgl. Josef Boyer / Helge Heidemeyer (Bearb.), Die Grünen im Bundestag. Sitzungsprotokolle und Anlagen 1983-1987, Düsseldorf 2008.

${ }^{7}$ Vgl. Josef Boyer, Die Grünen. Mitgliedschaft und Sozialstruktur, in: Ders./Till Kössler (Bearb.), Handbuch zur Statistik der Parlamente und Parteien in den westlichen Besatzungszonen und in der Bundesrepublik Deutschland. SPD, KPD und kleinere Parteien des linken Spektrums sowie die Grünen. Mitgliedschaft und Sozialstruktur 1945-1990, Düsseldorf 2005, S. 943-1032.

${ }^{8}$ Vgl. Silke Mende, „Nicht rechts, nicht links, sondern vorn“. Eine Geschichte der Gründungsgrünen, München 2011.

${ }^{9}$ Vgl. Saskia Richter, Die Aktivistin. Das Leben der Petra Kelly, München 2010.

${ }^{10}$ Vgl. Mende, Von der „Anti-Parteien-Partei“, in: Woyke (Hrsg.), Wandel des Politischen.

${ }^{11}$ Vgl. Regina Wick, Die Mauer muss weg - Die DDR soll bleiben. Die Deutschlandpolitik der Grünen von 1979 bis 1990, Stuttgart 2012.

${ }^{12}$ Ludger Volmer, Die Grünen und die Außenpolitik - ein schwieriges Verhältnis. Eine Ideen-, Programm- und Ereignisgeschichte grüner Außenpolitik, Münster 1998, das Zitat S. 273. Insgesamt spielt das Verhältnis zu Gorbatschow und zur Sowjetunion aber für ihn nur eine untergeordnete Rolle. 
damit vor der wirklichen Gefahr - der sich ständig schneller drehenden Rüstungsspirale - ablenkte ${ }^{13}$. Das bedeutete keine Verteidigung der östlichen Rüstungspolitik: Die Grünen vertraten hier mehrheitlich einen Ansatz der Äquidistanz, der sowjetische und amerikanische Rüstungsprogramme gleichermaßen verurteilte ${ }^{14}$.

Gleichwohl artikulierten die meisten Grünen damals „eine scharfe Amerikakritik“, wobei es vor allem um den „American way of life“ sowie die Sicherheits- und Außenpolitik der USA ging. Als ,antiamerikanisch“ wollten sie indes nicht gelten, da sie zwischen den friedlichen Bürgern der Vereinigten Staaten und deren kriegstreiberischen Führung unterschieden ${ }^{15}$. Die Kritik an der anderen Supermacht und den Ostblockstaaten entzündete sich dagegen an der Besetzung von Afghanistan und an Verstößen gegen die Menschenrechte. Hier solidarisierten sich die meisten Grünen mit den oppositionellen und dissidenten Gruppen, sei es in Polen, in der DDR, der ČSSR oder in der Sowjetunion ${ }^{16}$.

Über die Bedeutung der Menschenrechte in Osteuropa kam es Mitte der 1980er Jahre bei den Grünen zu einer Kontroverse. Petra Kelly und andere gingen, offensichtlich in Anlehnung an das Gedankengut der Charta 77, von der Unteilbarkeit von Frieden und Menschenrechten aus: Nur wenn in den Staaten selbst Frieden herrsche, also die Menschenrechte geachtet würden, sei auch „wahrer Friede" zwischen den Nationen möglich ${ }^{17}$. Gegen dieses Junktim wandten sich im Mai 1986 einige Grüne um Klaus Croissant und Dirk Schneider - beide übrigens Inoffizielle Mitarbeiter des Ministeriums für Staatssicherheit - mit dem Argument, man habe „angesichts der gesellschaftlichen Verhältnisse im westlichen Teil der Welt [...] nicht den geringsten Anlaß [...], die Situation der Menschenrechte West im Vergleich mit der Situation der Menschenrechte Ost als überlegen zu betrachten“18. Petra Kelly und ihre Unterstützer sahen darin eine Gefährdung des "grünen Grundkonsenses“, betonten jedoch, „daß innerer und äußerer Frieden zusammengehören“, und stellten fest, dass bestimmte Grundfreiheiten wie Pressefreiheit und Freizügigkeit „in der Tat ,bei uns‘ besser verwirklicht sind als in den

${ }^{13}$ Vgl. Susanne Schregel, Konjunktur der Angst. „Politik der Subjektivität“ und „neue Friedensbewegung“, 1979-1983, in: Bernd Greiner/Christian Th. Müller/Dierk Walter (Hrsg.), Angst im Kalten Krieg, Hamburg 2009, S. 495-520, hier S. 508-511; Judith Michel, „Richtige“ und „falsche“ Angst in der westlichen Debatte um den Nato-Doppelbeschluss, in: Patrick Bohrmann/Thomas Freiberger/Judith Michel (Hrsg.), Angst in den internationalen Beziehungen, Göttingen 2010, S. 251-272, hier S. $253 \mathrm{f}$.

${ }^{14}$ Vgl. Mende, Nicht rechts, S. 343 f.

15 Vgl. ebenda, S. 344-346.

${ }^{16}$ Vgl. ebenda, S. 342; Volmer, Die Grünen und die Außenpolitik, S. 274-277.

17 Vgl. Benjamin Müller, Von der Konfrontation zum Dialog. Charta 77, Menschenrechte und „Samtene Revolution“ in der Tschechoslowakei 1975-1989, in: Helmut Altrichter/Hermann Wentker (Hrsg.), Der KSZE-Prozess. Vom Kalten Krieg zu einem neuen Europa 1975 bis 1990, München 2011, S. 99-110, hier S. 104.

18 Klaus Croissant/Beno Hopmann/Barbara Lütkecosman/Angela Schäfers/Dirk Schneider, Zur Verknüpfung von Friedens- und Menschenrechtsfrage, in: Kommune. Forum für Politik, Ökonomie, Kultur 4 (1986), H. 5, S. 82-84, die Zitate S. 84. 
Staaten des realen Sozialismus“19. Letztlich blieb der Konflikt ungelöst, der vor allem in der Deutschlandpolitik eine wichtige Rolle spielte. Mit Blick auf die Sowjetunion gaben meist Kelly und ihre Getreuen den Ton an, wenngleich sich immer wieder Vertreter der anderen Gruppe zu Wort meldeten.

Bei den Grünen interessierten sich nur wenige für die Sowjetunion und Osteuropa. Neben Petra Kelly, für die dies jedoch nur ein Arbeitsgebiet unter vielen bildete, waren es vor allem die Mitglieder der Bundesarbeitsgemeinschaft Frieden, insbesondere aber die seit 1984/85 existierende Gruppierung „Ost-WestDialog“ (die oft auch als Arbeitskreis Ost-West-Fragen oder als Ost-West-AG firmierte $)^{20}$. Spiritus rector dieses Arbeitskreises war die promovierte Theaterwissenschaftlerin Elisabeth Weber, die zwischen 1970 und 1980 der maoistischen KPD angehört hatte, sich dann aber den Grünen zuwandte. Im Dezember 1983 wurde sie Mitarbeiterin der Grünen-Fraktion, wo sie besonders für die Abgeordneten Uli Fischer, Milan Horáček und Helmut Lippelt tätig war. Als ihr Markenzeichen galt das Engagement für die unterschiedlichsten Menschenrechtsgruppen in der DDR, Osteuropa und der Sowjetunion, zu denen sie auch persönliche Kontakte knüpfte. Freundschaftliche Beziehungen unterhielt sie etwa zur Frau des ausgebürgerten Schriftstellers Lew Kopelew, Raissa Orlowa, die Weber als Dozentin in ihrem Deutschkurs für Ausländer an der Universität Köln kennengelernt hatte ${ }^{21}$. Überdies waren bei offiziellen Anlässen oder Reisen die jeweiligen Sprecher und Sprecherinnen des Bundesvorstands und der Bundestagsfraktion qua Amt genötigt, sich hier zu engagieren.

Die folgenden Darlegungen gelten sowohl der Perzeption Gorbatschows durch die Grünen als auch den transnationalen Beziehungen zwischen dieser westdeutschen Partei und der sowjetischen Führung, den Dissidenten und den Oppositionellen. Dabei wird unter anderem danach gefragt, inwieweit das eigene Selbstverständnis sowie die Pflege oder die Vernachlässigung von Kontakten diese Perzeption prägten. Da auch das Bild von Gorbatschow und der Sowjetunion Wandlungen unterworfen war, orientiert sich der Beitrag, abgesehen vom ersten Abschnitt, in dem es um die Haltung der Grünen zu den Abrüstungsvorschlägen des sowjetischen Generalsekretärs bis 1987 geht, an der Chronologie. Nach ersten Begegnungen und Beurteilungen bis Ende 1986 kam es im Juni 1987 zu einer länger vorbereiteten Diskussion in der Bundestagsfraktion, die jedoch ohne greifbares Ergebnis blieb. Erst 1989 lebte die Gorbatschow-Debatte aufgrund seines spektakulären Abrüstungsvorschlags und seines Besuchs in der Bundesrepublik wieder auf. Im Laufe des Jahres 1990 mussten die Grünen erleben, wie sie mit ihrer ost- und deutschlandpolitischen Haltung schließlich im Abseits landeten.

\footnotetext{
${ }^{19}$ Uli Fischer/Milan Horacek/Petra Kelly/Elisabeth Weber, Was soll das Geholze? Croissant und andere gefährden leichtsinnig einen grünen Grundkonsens, in: Ebenda, H. 6, S. 58-60, die Zitate S. 58. Die Debatte wurde in den Heften 7 und 8 fortgesetzt.

${ }^{20}$ Vgl. Wick, Die Mauer muss weg, S. 168.

${ }^{21}$ Zur Biographie vgl. http://www.jugendopposition.de/index.php?id=2289 [20.2.2014]; Ehrung für Elisabeth Weber, in: Kölner Stadt-Anzeiger vom 2. 10.2003.
} 


\section{Die Grünen und die Abrüstungsvorschläge Gorbatschows 1985 bis 1987}

Schon bald nachdem Michail Gorbatschow am 11. März 1985 zum Generalsekretär des Zentralkomitees (ZK) der KPdSU gewählt worden war, beeindruckte er die westliche Welt nicht nur mit Umbesetzungen im Führungsapparat und ersten Reformankündigungen, sondern auch mit zahlreichen Rüstungskontroll- und Abrüstungsvorschlägen. Vor dem Hintergrund ihres Engagements für Abrüstung begrüßten die Grünen diese Initiativen uneingeschränkt. Anders als etwa die Experten vom Bundesinstitut für ostwissenschaftliche und internationale Studien in Köln hielten sie diese nicht für Propaganda, sondern für einen „ernstzunehmende[n] Ausdruck des Abrüstungswillens der Sowjetunion“22. Wie sehr sie sich in ihrer Ablehnung des Feindbilds Sowjetunion bestätigt sahen, verdeutlichte Fraktionssprecherin Annemarie Borgmann im Bundestag: „In dem Maße, in dem sich die sowjetische Bedrohungslegende in Verhandlungsangebote auflöst, entlarvt sich die westliche Notwehrpolitik mehr und mehr als rücksichtsloses Fallenkomplott. “23 Nicht die sowjetische, sondern die US-Regierung demonstriere etwa mit dem Festhalten an der Strategic Defense Initiative (SDI), dass sie kein Interesse an Abrüstungsvereinbarungen habe ${ }^{24}$.

Jedoch gab es nicht nur positive Stimmen zu den Abrüstungsinitiativen des KPdSU-Generalsekretärs. Das zeigt eine Kontroverse zwischen Wolfgang Bruckmann, einem Sicherheitsexperten und Mitarbeiter der Grünen-Fraktion, und Michael Schmid-Vöhringer vom Verein für Friedenspädagogik Tübingen, dem Mitautor einer Broschüre über die Geschichte der Friedensbewegung in der Bundesrepublik. Bruckmann schätzte den Vorschlag Gorbatschows vom 15. Januar 1986, bis 2000 etappenweise alle Kernwaffen abzuschaffen, trotz einiger offener Fragen „grundsätzlich positiv“ ein und sah nun den Westen „am Zuge“. SchmidVöhringer hingegen erkannte zwar den Willen Gorbatschows an, die Aufrüstungsspirale zu stoppen; aber dieser habe damit „noch keine grundsätzlich neue Politik eingeläutet“. Denn auch Gorbatschow beharre auf „beiderseitige Maßnahmen“ und behalte „das gefährliche Paritätsdenken“ bei. Daher sollte die Friedensbewegung „ihre Hoffnungen nicht auf eine neue sowjetische Politik richten“25. Zwar setzten die Grünen auch weiterhin auf Gorbatschow; doch es bleibt auffällig, wie

${ }^{22}$ Archiv Grünes Gedächtnis (künftig: AGG), Petra Kelly Archiv (PKA), Nr. 178, Presseerklärung Nr. 34/86, 16.1.1986: Grüne begrüßen Gorbatschow-Vorschlag.

${ }^{23}$ Deutscher Bundestag, Stenographischer Bericht, 10. Wahlperiode, 214. Sitzung am 25.4.1986, S. 16447.

${ }^{24}$ Vgl. etwa AGG, PKA, Nr. 1589, Pressemitteilung Nr. 134 o.D. [März 1986]: Zur Rede Gorbatschows auf dem Parteitag der KPdSU. Bei SDI handelte es sich um den Plan zum Aufbau eines weltraumgestützten Abwehrschirms gegen Interkontinentalraketen.

${ }^{25}$ AGG, PKA, Nr. 178, Wolfgang Bruckmann, Der Gorbatschow-Plan: Grundsätzlich positiv mit Problemen im Detail, 29.1.1986; AGG, PKA, Nr.1134, Michael Schmid-Vöhringer, Der Gorbatschow-Plan: Die Wende zur Abrüstung?, 4.4.1986. Vgl. ders./Uli Jäger, „Wir werden nicht Ruhe geben ...". Die Friedensbewegung in der Bundesrepublik Deutschland 19451982. Geschichte, Dokumente, Perspektiven, Tübingen 1982. 
sehr sie auf einseitige Abrüstung drängten. Dies geschah etwa in einem Brief der Fraktionssprecher an Gorbatschow vom 24. April 1986, in dem sie seinen Aufruf vom 18. April begrüßten, auch konventionell in Europa abzurüsten, darüber hinaus aber anmahnten, seinen Initiativen „durch tatsächliche Schritte einseitiger Abrüstung Kraft zu verleihen“26.

Ungeachtet dessen änderte sich nichts daran, dass die Grünen Gorbatschow einen größeren Abrüstungswillen attestierten als der US-Administration unter Präsident Ronald Reagan. Als Gorbatschow beim Gipfel von Reykjavik am 11./12. Oktober 1986 vorschlug, alle Mittelstreckenraketen in Europa abzuschaffen, Reagan aber im Gegenzug nicht auf SDI verzichten wollte, sahen die Grünen darin erwartungsgemäß einen „Beleg für die außenpolitische Beweglichkeit der Sowjetunion unter Gorbatschow"; die Verantwortung für das Scheitern des Treffens lag für sie „allein beim amerikanischen Präsidenten“27.

Der Streit zwischen den Befürwortern einseitiger Abrüstung und den Realpolitikern, die durch bilaterale Vereinbarungen zum Ziel kommen wollten, eskalierte im Frühjahr 1987, als die Verhandlungen über die Intermediate Range Nuclear Forces (INF) nach Gorbatschows Zugeständnis, nicht länger auf dem gleichzeitigen amerikanischen Verzicht auf SDI zu bestehen, deutliche Fortschritte machten. Der Koordinierungsausschuss der Friedensbewegung bat im Frühjahr die GrünenFraktion, im Bundestag den sofortigen Abzug der Mittelstreckenwaffen aus der Bundesrepublik zu verlangen. Die Fraktion lehnte dieses Ansinnen zunächst ab, gab aber nach entsprechendem Druck der Basis am 2. Juni nach. Bei der namentlichen Abstimmung über den Antrag zwei Tage danach enthielten sich jedoch sechs Grünen-Abgeordnete mit der Begründung, dass angesichts der beginnenden Verständigung der Supermächte auf bilaterale Abrüstung ein solcher Schritt nicht sinnvoll sei ${ }^{28}$. Dies zeigte die Zerrissenheit der Grünen in einer für sie zentralen Frage; die Perzeption Gorbatschows wurde davon freilich nur am Rande berührt.

Als nach der Unterzeichnung des INF-Abkommens in Washington am 10. Dezember $1987^{29}$ der Bundestag über eine Regierungserklärung dazu diskutierte,

${ }^{26}$ AGG, Bestand Annemarie Borgmann, Nr. 8, Borgmann, Hönes, Volmer an Gorbatschow, 24.4.1986. Eine Antwort, die auch auf einseitige Schritte der Sowjetunion verwies, wurde ihnen am 11.6. 1986 von Botschafter Juli Kwiziniski übermittelt, AGG, B.II.3, Nr. 1063, Aktenvermerk Borgmann, 16.6. 1986. Auch im Gespräch mit Politbüromitglied Hermann Axen vom 2.9.1986 drängten Grünen-Vertreter auf einseitige Abrüstungsschritte: vgl. Wick, Die Mauer muss weg, S. 212.

${ }^{27}$ Deutscher Bundestag, Stenographischer Bericht, 10. Wahlperiode, 238. Sitzung am 16.10.1986, S. 18323 (Borgmann, erstes Zitat); AGG, PKA Nr. 178, Pressemitteilung Nr. 664/86, 15.10.1986: Erklärung von Petra Kelly und Gert Bastian (zweites Zitat). Vgl. auch Bestand Annemarie Borgmann, Nr. 10, Pressemitteilung Nr. 656/86, o. D. [Oktober 1986], Abrüstungschancen vertan. Erklärung zu den Nicht-Ergebnissen von Reykjavik.

${ }^{28}$ Vgl. Volmer, Die Grünen und die Außenpolitik, S. $184 \mathrm{f}$.

${ }^{29}$ In dem Abkommen wurde vereinbart, alle landgestützten Mittelstreckenwaffen zu vernichten und diese Vernichtung wechselseitig zu überprüfen. Es bildete den Abschluss eines langen, durch den NATO-Doppelbeschluss vom 12.12. 1979 eingeleiteten Prozesses, der die Stationierung von Mittelstreckenwaffen in Europa vorsah, wenn die Sowjetunion nicht ihre Mittel- 
waren die Grünen in ihrem Lob Gorbatschows wieder einig. So machte der „Realo" Alfred Mechtersheimer darauf aufmerksam, dass die Sowjetunion im Vergleich zu den USA das Doppelte an Trägersystemen und das Fünffache an Sprengköpfen in den Vertrag einbringe. Das bedeute einen grundlegenden Wandel gegenüber der sowjetischen Politik unter Breschnew: Der „Schlüssel für die Veränderung [sei daher] nicht im Nachrüstungsbeschluß, sondern in den Änderungen in Moskau zu sehen“30. Angelika Beer, die in den 1970er Jahren dem Kommunistischen Bund angehört hatte, nutzte die Gelegenheit, um auf drei Hindernisse aufmerksam zu machen, die weiteren Rüstungsanstrengungen im Westen entgegenständen: leere Kassen, Gorbatschows Abrüstungsinitiativen und die Friedensbewegung. Und sie fügte hinzu: „Ihr solltet nicht unterschätzen, was für eine Angst die Etablierten vor einer Neuauflage der Stationierungsdebatte [von] 1983 haben." ${ }^{31}$ Zwar sollte sich die letzte Bemerkung mit Blick auf die Debatte um die Kurzstreckenraketen als zutreffend erweisen; ihre Argumentation implizierte aber auch die These, dass Friedensbewegung und Grüne gemeinsam mit Gorbatschow für die Abrüstungserfolge verantwortlich seien. Sehr viel deutlicher formulierte es Grünen-Sprecherin Jutta Ditfurth vom fundamentalistischen Flügel am 19. Januar 1988 in einem Gespräch mit dem sowjetischen Außenminister Eduard Schewardnadse: Es seien „das Neue Denken in der Sowjetunion und die westliche Friedensbewegung [gewesen,] die den Vertrag durchgesetzt und den amerikanischen Rambo bezwungen“ hätten ${ }^{32}$. Eine der führenden Grünen präsentierte also die Friedensbewegung zu einem Zeitpunkt als Bündnispartner Gorbatschows, als dieser seine Politik immer deutlicher auf die Bundesregierung ausrichtete, und zeigte damit, wie sehr sie die Rolle der Friedensaktivisten überschätzte.

\section{Hoffnungen und Enttäuschungen 1986/87}

Welche Richtung Gorbatschow 1985/86 innenpolitisch einschlagen würde, war für die Grünen - wie für alle anderen politischen Akteure in der Bundesrepublik - nur schwer einzuschätzen. So schrieb etwa Joscha Schmierer, der Chefredakteur der den Grünen nahestehenden Zeitschrift Kommune, im Juli 1985, dass die aus machtpolitischen Überlegungen heraus eingeleiteten Reformen vielleicht zu mehr Effektivität führen könnten; aber „unter emanzipatorischen Gesichtspunkten ist von der Reform des Reiches wenig, von seinem Zerfall viel zu erwarten“33.

streckenraketen vernichtete. Da die Verhandlungen mit der Sowjetunion 1983 scheiterten, wurde auf westlicher Seite „nachgerüstet“. Eine wesentliche Rolle spielte dabei die Bundesrepublik, die ab November 1983 aufgrund eines Bundestagsbeschlusses der Stationierung amerikanischer Mittelstreckenwaffen auf eigenem Gebiet zustimmte.

${ }^{30}$ Deutscher Bundestag, Stenographischer Bericht, 11. Wahlperiode, 49. Sitzung am 10. 12. 1987, S. $3419 \mathrm{f}$.

${ }^{31}$ Ebenda, S. $3431 \mathrm{f}$.

${ }^{32}$ AGG, PKA, Nr. 470, Bericht vom Gespräch Grüne-Schewardnadse am 19. 1. 1988.

33 Joscha Schmierer, Ein Reformator im Zentrum des Reiches?, in: Kommune 3 (1985), H. 7, S. 64 . 
Mit anderen Worten: Einschneidende Veränderungen erwartete er nicht von den Reformvorhaben Gorbatschows, sondern von deren nicht intendierten Effekten an der Peripherie des sowjetischen Machtbereichs. Ähnlich unsicher war sich Petra Kelly bei der Beurteilung des XXVII. Parteitags der KPdSU vom 25. Februar bis zum 6. März 1986, zu dem sie notierte: „Eine Einschätzung ist sehr schwierig. Die Expertenmeinung[en] gehen weit auseinander." ${ }^{34}$ Die Wirtschaftsreformen boten dem „Realo“ Eckhard Stratmann zufolge, der Anfang April 1986 von einer einwöchigen Reise aus der Sowjetunion zurückkehrte, ein „sehr uneinheitliches Bild": Auf der einen Seite gebe es starke Zentralisierungsbestrebungen auf gesamtstaatlicher Ebene, andererseits solle die Wirtschaft auf regionaler und betrieblicher Ebene weitgehend dezentralisiert werden, wobei „erhebliche Realisierungsdefizite" bestünden ${ }^{35}$.

Es waren jedoch weniger die Wirtschaftsreformen als der Krieg in Afghanistan und die anhaltenden Menschenrechtsverletzungen, die das durch die Abrüstungsinitiativen aufgehellte Bild der Sowjetunion aus Sicht der meisten Grünen wieder verdunkelte. Dies wurde deutlich, als die für Oktober 1985 geplante Moskau-Reise einer Grünen-Delegation am 17. September platzte, da die sowjetische Botschaft die Teilnahme des Abgeordneten Ulrich Fischer unter Verweis auf dessen „,illegalen“ Aufenthalt in Afghanistan im Herbst 1984“ ablehnte ${ }^{36}$. Fischer hatte „schwere Vorwürfe sowohl gegen die sowjetische Besatzungspolitik als auch gegen die Einmischung von Seiten der USA erhoben“. Da für die Grünen aber das „Eintreten für das Selbstbestimmungsrecht der Völker und die Menschenrechte unteilbar" war, verzichteten sie auf die Reise, wenngleich sie weiterhin Interesse am Dialog mit der Sowjetunion bekundeten ${ }^{37}$.

Fraktionssprecherin Hannegret Hönes und Petra Kelly forderten im Dezember 1985 überdies Gorbatschow in zwei Briefen auf, nicht länger Dissidenten zu verfolgen und politische Gefangene wie Juri Orlow und Anatoli Schtscharanski ${ }^{38}$ freizulassen. Bereits vor Gorbatschows Machtantritt hatten sie sich für ein Ende der Verbannung der prominenten Regimekritiker Andrei Sacharow und seiner Frau Jelena Bonner eingesetzt. Im Dezember setzte eine Gruppe grüner Abgeordneter dieses Engagement mit einer Erklärung fort, in der sie sich „im Wissen, daß äußerer und innerer Friede zusammengehören“, mit den Dissidenten in der Sowjetunion solidarisch erklärte. Zugleich verurteilte sie das System, das von seinen Bürgern Treuebekenntnisse durch Diffamierung Andersdenkender verlange, und bekundete dem Ehepaar Sacharow ihren Respekt. Hönes schrieb überdies an

\footnotetext{
${ }^{34}$ AGG, PKA, Nr. 1589, [Petra Kelly,] KPdSU-Parteitag, o. D. (Hervorhebung im Original).

35 AGG, PKA, Nr. 469, Pressemitteilung Nr. 217/86.

${ }^{36}$ AGG, B.II.3, Nr. 1063, Bundes- und Fraktionsvorstand der Grünen an das Komitee für Sicherheit und Zusammenarbeit [in Moskau], 24.9.1985; Chronologie - Reise in die Sowjetunion, o.D.

${ }^{37}$ Ebenda, Pressemitteilung Nr. 593/85, Grüne Fraktion sagt geplante Reise in die Sowjetunion ab, 24.9.1985.

${ }^{38}$ Orlow und Schtscharanski waren Physiker und Dissidenten in der Sowjetunion, die 1976 die Moskauer Helsinki-Gruppe mitbegründeten. Diese sollte die Einhaltung der Bestimmungen der KSZE-Schlussakte in der Sowjetunion überwachen.
} 
Gorbatschow, dass dessen Abrüstungsvorschläge angesichts der sowjetischen Afghanistan- und Menschenrechtspolitik unglaubwürdig seien (womit sie nicht implizierte, dass die Grünen diese Initiativen nur für taktisch motiviert hielten) ${ }^{39}$.

Ungeachtet dieser Kritik an Gorbatschow unternahm eine Delegation des Bundesvorstands der Grünen vom 13. bis zum 19. April 1986 auf Einladung des ZK der KPdSU eine Reise nach Moskau und Leningrad. Die Delegation, die aus den „Realos“ Lukas Beckmann und Norbert Kostede, den „Fundis“Jutta Ditfurth und Rainer Trampert sowie dem Dolmetscher Rolf Schälike bestand, sprach sowohl mit der Partei- und Staatsführung (außer mit Gorbatschow) als auch mit Mitgliedern der sogenannten Vertrauensgruppe ${ }^{40}$. Nach ihrer Heimkehr zogen sie eine weitgehend positive Bilanz: So zeugte der erste Dialog mit den verantwortlichen Funktionären zu ökologischen Fragen „vom großen Interesse gegenüber den alternativen Vorstellungen der Grünen“. Aus den Gesprächen mit Boris Jelzin, Andrei Gromyko und Michail Simjanin gewannen sie den Eindruck, dass die Abrüstungsvorschläge Gorbatschows ernst zu nehmen seien. Nur im Zusammenhang mit dem „Demokratieverständnis konnten die teilweise wesentlich unterschiedlichen Denkweisen und Erfahrungen nicht auf einen Nenner gebracht werden“. Zudem ergaben die Ergebnisse der Unterredungen mit Mitgliedern der „Gruppe zur Herstellung des Vertrauens zwischen der UdSSR und den USA“, kurz Vertrauensgruppe genannt, dass in der Sowjetunion „parallel zu einer merklich offeneren Atmosphäre [...] eine verschärfte Verfolgung von politisch Andersdenkenden erfolgt" ${ }^{\text {"41 }}$. Auf Trampert hatte der Stadtparteichef von Moskau, Boris Jelzin, einen vorwiegend negativen Eindruck gemacht: Dieser habe wie ein Manager gewirkt, für den die „Steigerung des Wachstums“ von zentraler Bedeutung sei und der „sehr populistisch“ vorgehe. Leute wie er versuchten, durch Säuberungen im mittleren Management und die Schaffung von Anreizen die Wirtschaft von oben zu erneuern. Die neue Entwicklungsphase in der Sowjetunion berühre daher „nicht die allgemeine gesellschaftliche Demokratie“42. Trampert konnte folglich den sowjetischen Wirtschaftsreformen nicht viel abgewinnen, da sie sich in ihrer Wachstumsfixierung nicht vom westlichen Modell unterschieden und auf eine Anpassung an die Marktwirtschaft hinauszulaufen schienen.

${ }^{39}$ AGG, PKA, Nr. 464, Hönes an Gorbatschow, o. D. [11.12.1985], Kelly an Tolkunow, 11. 12. 1985, Kelly an Gorbatschow, 11.12. 1985; AGG, PKA Nr. 465, Grüne: Den Kampf um Frieden mit dem Kampf um Menschenrechte verbinden, 10.12.1985 (Erklärung von Uli Fischer, Heinz Suhr, Petra Kelly, Otto Schily); Elisabeth Weber, Bericht über unsere Solidaritätsarbeit für A. Sacharow und J. Bonner für die AG Ost-West-Dialog, 1. 6. 1984.

${ }^{40}$ Die Vertrauensgruppe konstituierte sich 1982 und setzte sich zunächst für konstruktive Vorschläge zur Herstellung eines Vertrauensverhältnisses zwischen den Supermächten und später auch zwischen den Menschen aus Ost und West ein; vgl. dazu AGG, PKA, Nr. 468, Rolf Schälike, Die „Vertrauensgruppen“ in der Sowjetunion, in: osteuropa-forum Nr. 12, Mai 1986. Zu deren weiterer Entwicklung vgl. von Saal, KSZE-Prozess und Perestroika, S. 91 f. u. S. 103-105.

41 AGG, B.II.3, Nr. 1064, Bericht über die Delegationsreise des Bundesvorstandes der Grünen in die UdSSR vom 13.-19.4.1986.

${ }^{42}$ Ebenda, Grünen-Pressedienst, Nr. 45/86, Neuer Kurs in der Sowjetunion?, 21.4. 1986. 
Trotz dieser Einschränkungen schätzten die grünen Delegierten die Gespräche zur Friedens- und Sicherheitspolitik, zu Demokratie und Menschenrechten als „weitgehend konstruktiv und [...] informativ“ ein, auch wenn es Differenzen gegeben habe. Überdies bewerteten sie das „Interesse der KPdSU an einem Dialog mit den Grünen [...] als stabil und langfristig“. Dieses Interesse speise sich vor allem aus den „ernstgemeinten abrüstungspolitischen Absichten der KPdSU“, die nur mit Unterstützung der westeuropäischen Friedensbewegung umgesetzt werden könnten. Daraus ergab sich aus Sicht der Berichterstatter die Möglichkeit für die Grünen, „eigene Ziele, Inhalte und Formen in einen solchen Dialog einzubringen und umzusetzen"43. Die Grünen verkannten offensichtlich, dass sie für die sowjetische Führung nur von instrumentellem Wert waren: Moskau wollte die Grünen - genauso wie die SPD - nutzen, um sich im politischen Meinungsstreit in der Bundesrepublik Gehör und Einfluss zu verschaffen. Die Grünen waren in dieser Beziehung sicher eigensinnige und unbequeme Partner, aber sie überschätzten ihr Gewicht, wenn sie meinten, die sowjetische Politik langfristig beeinflussen zu können. Dies hatten sie aber zweifellos im Sinn, da sie mit der internationalen Abteilung der KPdSU Vereinbarungen zum Informations-, Artikel-, Besucherund Referentenaustausch sowie zur Förderung von Städtepartnerschaften schlos$\operatorname{sen}^{44}$.

Kurz nach der Rückkehr der Grünen-Delegation, am 26. April 1986, explodierte Block 4 des Kernkraftwerks Tschernobyl. Die Grünen machten die Reaktorkatastrophe umgehend zum Thema, um die sofortige Abschaltung aller Atomkraftwerke in der Bundesrepublik zu erreichen. Im Unterschied zum Bundesvorstand und zu den Fraktionssprechern übten einzelne Landesverbände durchaus Kritik an Gorbatschow. So schrieb etwa Wolfgang Schenk für die Fraktion der Alternativen Liste im Berliner Abgeordnetenhaus einen offenen Brief an den Generalsekretär, in dem er Mitgefühl für die Opfer und „Empörung und Bestürzung über das verantwortungslose Handeln der sowjetischen Behörden“ äuBerte; die sowjetische Informationspolitik bezeichnete er als „Ausdruck menschenverachtenden Denkens und Handelns“"45. Einige Grüne trafen Anfang Mai mit Jelzin zusammen, der zum Parteitag der Deutschen Kommunistischen Partei (DKP) angereist war. Jelzin, der die westdeutsche Presse wegen Verbreitung „freche[r] Lügen“ über die Reaktorkatastrophe öffentlich scharf angegriffen hatte, wiegelte gegenüber den Grünen ab. Im Rückblick beschrieben diese den Tenor seiner Äußerungen wie folgt: „Was ist denn schon passiert? Was regt ihr euch auf? Glaubt eurer Bundesregierung! “46 Diese Äußerungen wurden zwar als unbefriedigend empfunden, aber erst am 13. Oktober beschloss der Fraktionsvor-

\footnotetext{
${ }^{43}$ Ebenda, Bericht über die Delegationsreise des Bundesvorstandes der Grünen in die UdSSR vom 13.-19.4. 1986 (Hervorhebungen im Original).

${ }^{44}$ Ebenda, Norbert Kostede, Vereinbarungen zwischen den Grünen und der Internationalen Abteilung beim ZK der KPdSU, 21.4.1986.

45 AGG, B.II.3, Nr. 1063, Schenk an Gorbatschow (Offener Brief), 30. 4. 1986.

${ }^{46}$ AGG, B.II.3, Nr. 1064, Bericht über die Delegationsreise des Bundesvorstands und der Bundestagsfraktion der Grünen in die UdSSR vom 11.-14.11.1986; das Zitat von Jelzin in: „Die „Hauptfront des Klassenkampfes“ und Tschernobyl“, in: Frankfurter Allgemeine Zeitung vom
} 
stand mit drei gegen zwei Stimmen, den offenen Brief einer Bürgerinitiative an Gorbatschow „zur bestürzenden Wiederaufnahme des Betriebs des Atomkraftwerkes in Tschernobyl mit zu unterzeichnen“47. Diese starke Zurückhaltung ist wahrscheinlich darauf zurückzuführen, dass die Grünen antisowjetischen Stimmungen bei sich keinen Raum geben und nicht in die Kritik vom rechten Flügel des politischen Spektrums einstimmen wollten. Dessen ungeachtet waren sie von Gorbatschow enttäuscht. Norbert Kostede vom Bundesvorstand vertrat die Auffassung, dass international drei Hauptakteure die „Rüstungseskalationspolitik der Reagan-Administration und deren Verbündeter“ bekämpften: „Blockfreie, Friedensbewegung, Sowjetunion“. Nach Tschernobyl habe sich die "Situation der internationalen Abrüstungskoalition“ verschlechtert, weil „die Sowjetunion eine Doppelrolle spielt und sich zugleich im gegnerischen Lager herumtreibt: in der internationalen Atomkoalition“. Der Spielraum eines Dialogs zwischen der Sowjetunion und den Grünen verenge sich daher; gleichwohl würden die Grünen auch nach Tschernobyl „hartnäckig an die ,Dialog"-Vereinbarungen erinnern" ${ }^{4}$.

Weil von Seiten der Grünen nach wie vor erhebliches Interesse an der Fortsetzung der Gespräche mit der Sowjetunion bestand, nahmen sie eine Einladung des sowjetischen „Komitees für Europäische Sicherheit“ nach Moskau an. Diesmal bestand die Delegation aus den „Realos“ Otto Schily, Norbert Kostede, Lukas Beckmann und den „Fundis“Jutta Ditfurth und Annemarie Borgmann sowie aus dem Pressesprecher Franz Stänner und dem Dolmetscher Rolf Schälike. Am ersten Tag des Besuchs, dem 11. November 1986, bekundeten sie in einem Schreiben an Gorbatschow unter anderem ihre Hoffnung, über einen Ausstieg aus der Atomenergie zu sprechen. Die prominentesten Gesprächspartner waren diesmal Andrei Gromyko, Eduard Schewardnadse und Anatoli Dobrynin; die Grünen führten aber auch „Basisgespräche“ mit der Vertrauensgruppe. Die Urteile über die sowjetische Politik fielen weniger positiv aus als im Frühjahr. Kritik an Atomenergie sei inzwischen zwar „nicht mehr tabu“ - und die Grünen konnten das Thema, anders als im April, auch ansprechen. Aber die sowjetische Führung halte an ihrer Atomenergiepolitik fest; es sehe sogar so aus, „als ob die Katastrophe von Tschernobyl ein Symbol zur Überwindung unvermeidlicher Schwierigkeiten geworden ist, daß Tschernobyl die Kräfte in Richtung einer pro-westlichen Industriepolitik mobilisiert und fördert". Auch in der Sicherheits- und Außenpolitik waren die Berichterstatter ziemlich unzufrieden mit den sowjetischen Antworten. So äußerten sie den Verdacht, dass die kurz zuvor gescheiterten Gespräche in Reykjavik möglicherweise „nur propagandistischen Zielen gedient“ hätten und dass auch das sowjetische „Bestehen auf dem SDI-Verbot [...] nicht 100-prozentig“

5.5. 1986. Vgl. auch das Interview mit Jelzin, „Vielleicht war nur ein Mensch schuld“, in: Stern vom 7.5. 1986.

${ }^{47}$ Protokoll über die 96. Sitzung des Fraktionsvorstandes, 13.10.1986, Teil 2, in: Boyer/Heidemeyer (Bearb.), Die Grünen im Bundestag, S. 973 (für den offenen Brief vgl. Anlage A, S. 974).

${ }^{48}$ Norbert Kostede, Internationale Politik nach Tschernobyl. Zur Delegationsreise des Bundesvorstandes der Grünen nach Moskau und Leningrad, in: Kommune 4 (1986), H. 6, S. 28-31, hier S. $28 \mathrm{f}$. 
sei. Die Äußerungen zu einem blockfreien Europa - einer Lieblingsidee von Otto Schily ${ }^{49}$ - blieben zudem „sehr allgemein und unkonkret“, so dass hier festgehalten wurde: „Die heutige Position der Sowjetunion konnte nicht überzeugen.“ Differenzen bestanden des Weiteren bei Menschenrechtsfragen. Trotz größerer Offenheit der sowjetischen Führung sei die Entwicklung weiterhin widersprüchlich: „Wir erfuhren von mehr Demokratie, Offenheit, aber auch von verstärkten Verfolgungen." Obwohl im Gesamturteil die Reise als „sinnvoll“ bewertet wurde, warnten die Verfasser des Abschlussberichts davor, deren Bedeutung zu überschätzen ${ }^{50}$.

Gleichwohl waren die Grünen davon überzeugt, dass die sowjetische Seite sie als Partner für ihre Außen- und Abrüstungspolitik benötige. Norbert Kostede brachte dies in einem Artikel für die Kommune auf die selbstironische Formel: „Roter Bär sucht grünen Gartenzwerg. Daß dieser Wicht recht streitsüchtig ist [...], nimmt man als Risiko in Kauf." ${ }^{\text {51 }}$ Die offiziellen sowjetischen Gesprächspartner vergaßen auch nicht, dem grünen Selbstbewusstsein zu schmeicheln, indem sie mehrfach betonten, dass deren Vorschläge „genauestens diskutiert“ würden. Die Berichterstatter über die Reise vom November 1986 waren zwar realistisch genug, um abschließend festzuhalten: „Das Gewicht der Grünen in der Sowjetunion hängt im wesentlichen von ihrer Bedeutung in der Bundesrepublik Deutschland, von einer erfolgreichen grünen Politik ab. “52 Daran, dass die Sowjetunion die Grünen für einen wichtigen Partner in der Bundesrepublik hielt, hatten sie aber keinen Zweifel.

Die Reise sollte ein parteiinternes Nachspiel haben. Fraktionssprecherin Annemarie Borgmann echauffierte sich in einem internen Schreiben vom 1. Dezember 1986, das fast vollständig im Spiegel abgedruckt wurde, zum einen über die Zusammensetzung der Delegation und zum anderen darüber, dass jedes Delegationsmitglied versucht habe, „mit seinem Steckenpferd die Gespräche zu dominieren“, ohne vorher eine gemeinsame Linie abgesteckt zu haben. Mit drastischen Worten schrieb sie daher: „Der Brachialo-Flügel in Moskau: mir war elend und zum Kotzen zumute!“ Doch Borgmann beließ es nicht bei gekränkter Eitelkeit; sie erhob auch taktisch-inhaltliche Einwände gegen eine zu heftige Kritik an der sowjetischen Rüstungskontrollpolitik, wie sie vor allem von Beckmann „gegenüber derjenigen Blockmacht [vorgetragen worden sei], die von den Grünen im Bundestag mehr denn je als Hoffnungsträger für einen Ausstieg aus der Rüstungsspirale angesehen wird"53. Daraus lässt sich entnehmen, dass sie den sowjetischen

\footnotetext{
${ }^{49}$ Vgl. Otto Schily, Perspektiven der Friedenspolitik: Die „Mitteleuropäische Friedensunion“, in: Ders., Vom Zustand der Republik, Berlin (West) 1986, S. 103-110.

${ }^{50}$ AGG, B.II.3, Nr. 1064, Bericht über die Delegationsreise des Bundesvorstands und der Bundestagsfraktion der Grünen in die UdSSR vom 11.-14.11.1986.

${ }^{51}$ Norbert Kostede, Gorbatschows Risiko, in: Kommune 5 (1987), H.1, S. 38 f., hier S. 38.

52 AGG, B.II.3, Nr. 1064, Bericht über die Delegationsreise des Bundesvorstands und der Bundestagsfraktion der Grünen in die UdSSR vom 11.-14. 11. 1986.

${ }^{53}$ AGG, PKA, Nr. 2540, Annemarie Borgmann an Bundestagsfraktion, Bundesvorstand, Bundeshauptausschuss, BAG Frieden, BAG Internationalismus, 1.12.1986; dies., Der Brachialo-Flügel in Moskau, in: Der Spiegel vom 15. 12. 1986. Norbert Kostede reagierte darauf im
} 
Kurs sehr viel stärker unterstützte als etwa der „Realo“ Beckmann; da sie sich nicht hatte durchsetzen können, zog sie sich mit dem Ende der Legislaturperiode aus dem Bundestag zurück.

Trotz der gewachsenen Skepsis gegenüber Gorbatschow unter zahlreichen grünen „Ostpolitikern“ rissen die Kontakte zwischen den Grünen und der sowjetischen Führung nicht ab. Auch Petra Kelly und Gert Bastian bemühten sich in einem Brief im Dezember 1986 um eine Audienz beim sowjetischen Parteichef. Sie wollten Gorbatschow ihre Sorgen über ausbleibende Abrüstungsvereinbarungen vortragen und die Gruppe „Generale für den Frieden“ vorstellen, als deren deutscher Sprecher der ehemalige Bundeswehrgeneral Bastian fungierte ${ }^{54}$. Im Februar 1987 traten beide in Moskau auf, wo Petra Kelly auf dem Friedensforum sprach, einer Großveranstaltung mit 900 Teilnehmern aus aller Welt. Vor ihrer Reise erlebten die Grünen ein Wechselbad der Gefühle. Am 8. Dezember starb der prominente Regimegegner Anatoli Martschenko in Haft, was die Grünen zu einem offenen Brief an Gorbatschow veranlasste, in dem sie diesen um Freilassung aller politischen Gefangenen und um Auflösung der psychiatrischen Sonderkliniken und Straflager baten ${ }^{55}$. Nur wenige Tage später, am 16. Dezember, erklärte Gorbatschow die Verbannung Sacharows und Bonners für aufgehoben, was nicht nur die Grünen als „ein Zeichen der Hoffnung [werteten], daß sich der Demokratisierungsprozess in der Sowjetunion positiv entwickelt" ${ }^{\star 56}$. Unmittelbar danach traten Kelly und Bastian in einen Meinungsaustausch mit Sacharow ein, den sie bei ihrem Moskau-Aufenthalt fortsetzten ${ }^{57}$.

Zum Abschluss des Friedensforums am 16. Februar $1987^{58}$ hatten die beiden grünen Spitzenpolitiker Gelegenheit, mit Gorbatschow persönlich zu sprechen. Sie übergaben einen Bericht von Amnesty International zur Lage der politischen Gefangenen in der Sowjetunion und dankten für die Rückkehr des Ehepaars Sacharow nach Moskau, während Gorbatschow zum Erfolg der Grünen bei den Bundestagswahlen vom Januar gratulierte. Als der Generalsekretär schließlich „auch noch einen Button mit dem Bild ,Schwerter zu Pflugscharen“" entgegennahm, war Petra Kelly begeistert. Zu einem Journalisten sagte sie: „Ich bin ja wirklich nicht leicht umzuhauen, aber das hat mich umgehauen. " ${ }^{59}$ In einer Pressemit-

Grünen-Pressedienst (Nr. 189/86) am 17.12.1986 unter anderem mit der Aussage, dass ihm „von Annemarie Borgmann kein eigenständiger und qualifizierter Beitrag zur Abrüstungsund Friedenspolitik bekannt" sei, in: AGG, PKA, Nr. 2540.

54 AGG, PKA, Nr. 2540, Kelly und Bastian an Gorbatschow, 8. 12. 1986.

${ }^{55}$ Ebenda, Pressemitteilung Nr. 813/86, Offener Brief an Gorbatschow anläßlich des Todes von Anatoli Martschenko, 10.12.1986.

56 AGG, PKA Nr. 465, Pressemitteilung Nr. 844/86, Erklärung zur Aufhebung der Verbannung Sacharows, 19.12.1989.

57 AGG, PKA, Nr. 2113, Kelly und Bastian an Sacharow, 5. 2. 1987; AGG, PKA, Nr. 2537, Pressemitteilung Nr. 84/87, Petra Kelly und Gert Bastian tief beeindruckt von Gespräch mit Andrej Sacharow und Jelena Bonner, o. D. [Februar 1987].

58 Kelly referierte zum Zusammenhang von „Frieden, Abrüstung, Umweltschutz und Menschenrechten“. AGG, PKA, Nr. 2537, Beitrag von Petra Kelly auf dem Moskauer Friedensforum am 14.2.1987.

${ }^{59}$ Johannes Grotzky, Das Ergebnis einer neuen Denkweise, in: Die Zeit vom 20. 2. 1987. 
teilung nach ihrer Rückkehr formulierten Kelly und Bastian: „Die Grünen zeigten sich beeindruckt von der Persönlichkeit des Generalsekretärs Gorbatschow, den sie als ,unglaublich offen, gradlinig und warmherzig" empfunden hatten." ${ }^{60}$ Die Beziehungen zwischen den Grünen und der Sowjetunion unter Gorbatschow erhielten damit eine persönlich-emotionale Dimension, die für Petra Kelly, die ihre Gefühle sehr ernst nahm ${ }^{61}$, von besonderer Bedeutung werden sollte. Gorbatschow hatte mit seiner Gewandtheit und Spontaneität dazu beigetragen; dass seine Offenheit und seine geschickten Gesten verfingen, war jedoch wesentlich auf die Persönlichkeit von Petra Kelly zurückzuführen.

\section{Wohin treibt die Sowjetunion? Debatten und Auseinandersetzungen 1987}

Anlass zu einer systematischeren Meinungsbildung über Gorbatschow und seinen Kurs unter Beteiligung der Bundestagsfraktion war ein von der Bundesarbeitsgemeinschaft (BAG) Frieden im Herbst 1986 geplanter Kongress zum Thema „Sowjetunion auf dem Prüfstand“, um den sich eine am 25./26. Oktober eingesetzte Vorbereitungsgruppe kümmern sollte ${ }^{62}$. Elisabeth Weber war maßgeblich daran beteiligt, indem sie Materialien zusammenstellte und verschickte. Zwar diskutierte die BAG Frieden darüber im Februar 1987; aber obwohl Kostede darauf bestand, „daß ein SU-Kongreß auf jeden Fall stattfinden werde“, verfolgte man die Idee nicht weiter ${ }^{63}$. Dafür machte sich Ende Februar Elisabeth Weber „Gedanken über eine Diskussion der Fraktion zum Thema der Reformpolitik von Gorbatschow"64. Hintergrund war höchstwahrscheinlich das im Westen breit rezipierte Januarplenum des ZK der KPdSU, auf dem Gorbatschow erbarmungslos mit der Breschnew-Ära abrechnete und „Demokratisierung“ forderte - insbesondere in der KPdSU. Eine Sondersitzung des Arbeitskreises Abrüstung, Frieden und Internationales (AFI) mit auswärtigen Experten am 4. Mai diente der Vorbereitung der Fraktionssitzung, die schließlich am 16. Juni stattfand. Weber und ihre Mitstreiter verfolgten damit mehrere Ziele: Erstens wollten die Grünen in der neuen sowjetischen Öffentlichkeit mehr und andere Gesprächspartner finden; zweitens sollte eine Grundlage geschaffen werden, um in der westdeutschen Diskussion über die Sowjetunion neue Impulse geben zu können, doch dazu musste - drittens - die Ostpolitik bei den Grünen insgesamt ein höheres Gewicht bekommen $^{65}$.

\footnotetext{
${ }^{60}$ AGG, PKA, Nr. 2537, Pressemitteilung Nr. 102/87, Petra Kelly und Gert Bastian treffen Gorbatschow, 17.2.1987.

${ }^{61}$ Vgl. Richter, Die Aktivistin, S. 277-280.

${ }^{62}$ Das geht aus einer Bemerkung von Harald Hellweg-Mahrt hervor; ders., Gedanken zur bisherigen „Sowjetunion-Diskussion“ in der BAG Frieden, 2.3.1987, in: AGG, B.II.3, Nr. 1033.

${ }^{63}$ Ebenda, Protokoll der BAG Frieden, o.D., [Februar 1987].

${ }^{64}$ AGG, PKA, Nr. 469, Weber an Fraktionsvorstand (Kelly, Lippelt, Schily), 28. 2. 1987.

${ }^{65}$ Ebenda, Elisabeth Weber, Thesen zum Verhältnis Grüne-Gorbatschows Reformpolitik, Entwurf, 15.3.1987.
} 
Elisabeth Weber spielte in dieser Debatte eine wichtige Rolle, da sie eine der wenigen Experten war, die sich bereits vor 1985 stärker mit der Sowjetunion und Osteuropa befasst hatten. Ein langes Papier, das sie am 18. Januar 1987 zur Vorbereitung des Kongresses an die Teilnehmer der BAG Frieden verschickte ${ }^{66}$, enthielt unter anderem Äußerungen zu den Systemen in Osteuropa, zur Sowjetunion und zu den dortigen Oppositionellen. Die realsozialistischen Systeme betrachtete sie mit Skepsis: Ein „Reich des Bösen“ (so Ronald Reagan) war die Sowjetunion und ihr Imperium für sie zwar nicht, aber die dortigen Gesellschaften hielt sie für instabil. Die Sowjetunion verfolge keinen „Plan einer militärischen Eroberung und Besetzung Westeuropas“, aber als „Friedensmacht“ könne sie aufgrund ihres riesigen Militärpotenzials und des Stellenwerts, den das Militär dort besitze, auch nicht gelten. Die demokratischen Oppositionsgruppen in Osteuropa, die die Grünen „als unsere authentischen Verbündeten“ ansahen, träten dort „für die gesellschaftliche Erneuerung ihrer Systeme“ ein. Doch seien deren zentrale Themen weder Frieden noch Umweltschutz noch Emanzipation der Frau, sondern es gehe vielmehr um „die Frage nach einer demokratischen Gesellschaft“. Dazu „sollten Grüne sich [nicht] zynisch oder überheblich verhalten“, sondern sich „trotz aller negativen Erfahrungen mit dem Staatsapparat der BRD“ zu Gewaltenteilung und Rechtsstaat als Grundlage der eigenen „Vorstellung von Staat und Gesellschaft [und] Grundlage des Nachdenkens über weitere Demokratisierung“ bekennen. Diese Formulierungen zeigen bereits, welche Schwierigkeiten viele Grüne mit osteuropäischen Oppositionellen hatten: Aufgrund ihrer oft ins Fundamentalistische gehenden Opposition zur Bundesrepublik war ihnen unverständlich, wie Dissidenten in Osteuropa in den westlichen Systemen ein Vorbild sehen konnten. Elisabeth Weber unterschied sich aufgrund ihrer persönlichen Kontakte zu Oppositionellen in Osteuropa darin von zahlreichen ihrer grünen Mitstreiter.

In ihrer Februar-Sitzung und in zwei Papieren kritisierten die Mitglieder der BAG Frieden Webers Vorlage „unter mehreren Gesichtspunkten“67. Dabei wollte Magistratsdirektor Alexander Schubart aus Frankfurt am Main vor allem den geplanten Kongress-Titel ändern, er plädierte für eine Veranstaltung „mit und nicht über die Sowjetunion“. Zur Demokratie- und Menschenrechtsproblematik bemerkte er, dass „es keinen Staat der Welt gibt - insbesondere auch nicht die BRD selbst - in dem die Gewährleistung der Menschenrechte widerspruchlos und bruchlos gegeben wäre“. Es gehe lediglich „um einen Normal- und Mindeststandard an Menschenrechten“, auf dem man bestehen müsse; die Existenz von konkurrierenden politischen Parteien gehöre aber nicht dazu ${ }^{68}$. Harald HellwegMahrt hingegen kritisierte das Papier sehr viel grundsätzlicher und verband mit

${ }^{66}$ AGG, B.II.3, Nr. 1033, Weber an die Teilnehmer der BAG Frieden, 18. 1. 1987; das 20-seitige Papier trägt keinen Titel und war Anfang 1985 im AFI erarbeitet worden.

${ }^{67}$ Ebenda, Protokoll der BAG Frieden, o.D., [Februar 1987]. Das Protokoll, das Weber am 18.3.1987 an Clasen, Schälike, Süß und Timm verschickte und als „nicht sehr freundlich“ bezeichnete, ist allerdings sehr allgemein gehalten und enthält so gut wie keine Äußerungen, die einzelnen Teilnehmern zugeordnet werden können.

${ }^{68}$ Ebenda, Schubart an BAG Frieden, 12. 2. 1987. 
seinen Bemerkungen auch eine implizite Gorbatschow-Kritik. So fragte er etwa, ob die „,Reaktivierung“ Lenins als Idol“ nicht vor dem Hintergrund von „Gorbis Disziplinforderungen zu verstehen“ sei, mit dem „Ziel, zu einem ,modernen intensiven Wirtschaftswachstum“ zu gelangen“. Die Industriegesellschaft schaffe, unabhängig vom politischen System, „automatisch unpersönliche Arbeitsstrukturen und nicht minder unpersönliche, unbarmherzige und mechanische menschliche Beziehungen. Diese Strukturen bedingen den bürokratischen Staat mit seinen Verwaltern, Moralisten, Richtern, Psychiatern und Umerziehungslagern!" Vor diesem Hintergrund sei es ihm völlig unverständlich, wie die Osteuropäer „das westliche System als das ,bessere“ ansehen“ könnten ${ }^{69}$. Da Grüne wie Hellweg-Mahrt die moderne Industriegesellschaft letztlich als Grund allen Übels identifizierten, verlor für sie der zentrale Unterschied zwischen Demokratie und Diktatur an Bedeutung, auf dem die osteuropäischen Oppositionellen vor dem Hintergrund eigener bitterer Erfahrungen beharrten.

Auch mit ihren „Thesen zum Verhältnis Grüne-Gorbatschows Reformpolitik“ versuchte Weber die Diskussion im Vorfeld der geplanten Fraktionssitzung zu steuern. Es handelte sich um eine Analyse, die sich weitgehend im Einklang mit dem westdeutschen Mainstream befand. Gorbatschows Versuch einer Reform von oben sei zunächst nur auf die Wirtschaft ausgerichtet gewesen; seit dem Januarplenum habe diese aber auch eine politische Dimension. Mit seiner Politik von Glasnost-also von Transparenz und Offenheit, insbesondere in den Medien - und der Freilassung politischer Gefangener habe er zwar die Intelligenzija gewonnen, nicht aber das entscheidende Konsumproblem gelöst: „Es ist viel leichter, die Schubladen der Dichter zu leeren“, zitierte Weber ihre Freundin Raissa OrlowaKopelewa, „als die Regale der Geschäfte zu füllen.“ Deshalb und auch wegen der gestiegenen Anforderungen an die Arbeitsdisziplin blieben die Arbeiter skeptisch. Gleichwohl verteidigte Weber Gorbatschow gegenüber Kritikern aus den Reihen der Grünen, die dessen „Politik als technokratische und autoritäre Reform“ verdammten. Immerhin setze er „einen Veränderungsprozeß in Gang [...], in dem sowohl unabhängige Positionen entstehen oder gestärkt werden können, die uns wesentlich näher stehen"70. Auch Weber sah offensichtlich in Gorbatschows Zielen kein Ideal; sie setzte aber auf gesellschaftliche Eigendynamik als Folge seiner Initiativen.

Mit diesen Thesen und anderem Material versehen, trafen sich Vertreter des Fraktionsvorstands und des Arbeitskreises Abrüstung, Frieden, Internationales am 4. Mai mit den Osteuropa-Historikern Wolfgang Eichwede und Michal Reiman, den Politikwissenschaftlern Klaus Segbers und Mária Huber, dem ehemaligen tschechischen Politiker und Gorbatschow-Bekannten Zdeněk Mlynár̆, dem Publizisten Gerd Koenen, Raissa Orlowa-Kopelewa, den Übersetzern Bernhard Clasen und Rolf Schälike sowie der Slawistik-Studentin Susanne Nies im Haus

\footnotetext{
${ }^{69}$ Ebenda, Harald Hellweg-Mahrt, Gedanken zur bisherigen „Sowjetunion-Diskussion“ in der BAG Frieden, 2.3.1987.

${ }^{70}$ AGG, PKA, Nr. 469, Elisabeth Weber, Thesen zum Verhältnis Grüne-Gorbatschows Reformpolitik, Entwurf, 15.3.1987.
} 
Wittgenstein bei Bonn, der Bildungsstätte der Grünen. Die Diskussion erbrachte zwar einen Konsens darüber, dass Gorbatschow mehr als nur eine technokratische Reform im Sinn habe; aber insbesondere die Gäste und Experten fanden auch genügend Kritikpunkte. Dabei äußerte sich Orlowa-Kopelewa am schärfsten: Man höre von Gorbatschow „immer schöne Worte“, sehe aber keine Taten. Mlynář bemängelte nicht nur das Fehlen eines „umfassenden Konzept[s] in der Führung“, sondern auch mangelnden Druck von unten. In eine ähnliche Richtung ging Eichwede, der konstatierte, Staat und Gesellschaft seien „auf die neue Situation nicht vorbereitet“. Schälike prognostizierte, die Reform werde „durch ein tiefes Tal führen“; die Grünen müssten wissen, dass die Sowjetunion „keine neue Gesellschaft, kein 3. Weg" sei - hier schwang die Warnung mit, in Gorbatschow nicht das hineinzuprojizieren, was dieser gar nicht beabsichtige ${ }^{71}$. Insgesamt waren daher die Erwartungen an die sowjetische Zukunft gedämpft, wenngleich man Gorbatschow den Willen zu tiefgreifenden Veränderungen bescheinigte.

Derart vorbereitet, konnte nach mehreren Verschiebungen die Fraktionssitzung über Gorbatschow am 16. Juni 1987 stattfinden, die mit Einleitungsstatements von Helmut Lippelt und Waltraud Schoppe begann. Petra Kelly sollte ebenfalls sprechen, konnte aber nicht teilnehmen. Ihre schriftlich überlieferten Thesen waren von der ihr eigenen Emotionalität geprägt. Gorbatschow, so ihre Überzeugung, handele bei seinen Reformen nicht aus taktischem Kalkül, sondern sei ein zutiefst glaubwürdiger, warmherziger und „von seiner Aufgabe überzeugter Mensch, der Vertrauen verdient“. So habe sie ihn auf dem Moskauer Friedensforum im Februar erlebt; auch „skeptischste Gesprächspartner haben später geäußert, wie sehr sie von Gorbatschows Glaubwürdigkeit und Gradlinigkeit beeindruckt waren“. Ihr Gefühl und die Konversion von Skeptikern zu Anhängern des Generalsekretärs waren für ihre Urteilsbildung maßgebend. Daraus ergab sich alles Weitere: Gorbatschow müsse seine Ausstrahlung nun auch gegen seine Widersacher im Apparat einsetzen, er müsse durch westliches Entgegenkommen gestützt werden, um ihm die außenpolitischen Erfolge zu bescheren, von denen er innenpolitisch abhänge. Seine neue Politik böte die „vielleicht einmalige Chance zur grundlegenden Neugestaltung des Ost-West-Verhältnisses“, die unbedingt zu nutzen sei, nicht zuletzt zur Verbesserung der Menschenrechtssituation im Ostblock. Kritisch sah sie lediglich, dass der sowjetische Kurs der Öffnung nach Westen auch eine Amerikanisierung der sowjetischen Konsumkultur mit sich brachte, wie sie sich etwa in der Eröffnung einer Filiale von McDonald's auf dem Roten Platz zeigte ${ }^{72}$.

Der eher moderate, von der SPD kommende Helmut Lippelt betonte zwar auch, „daß in der Sowjetunion ein Prozeß in Gang gekommen ist, der uns alle angeht“. Aber er argumentierte sehr viel rationaler, etwa mit den Erfahrungen, die Gorbatschow gemacht habe. Dabei waren diese Überlegungen nicht frei von

${ }^{71}$ AGG, B.II.1, Nr. 5177, Elisabeth Weber, Gesprächsnotiz von einer Diskussion über die Politik Gorbatschows am 4.5.1987 im Haus Wittgenstein, 17.5.1987.

${ }^{72}$ AGG, PKA Nr. 469, Petra Kelly, Betr.: Thesen zur Gorbatschow-Debatte in der Fraktion Die Grünen, Juni 1987. 
Spekulationen, so etwa, dass zu dessen „formativen Erlebnissen“ die OstblockKrisen von 1956, 1968 und 1980/81 gehörten. Aber er führte auch „belegbare Taten" an, die seiner Meinung nach die Ernsthaftigkeit von Gorbatschows Reformbemühungen zeigten: die Neubelebung rechtsstaatlicher Grundsätze, die Perestroi$k a$ - also der Umbau von Staat und Gesellschaft - und das Bündnis mit der Intelligenz. Er bewertete dies alles positiv, wie auch die von Gorbatschow in Gang gesetzte „Demokratisierung“, die „stärker auf institutionelle Absicherung von Prozessen [ziele], die die SU an die wirtschaftliche, aber auch kulturelle Entwicklung der Gegenwart wieder heranbringen soll“73.

Waltraud Schoppe schließlich trug ebenfalls eine weitgehend sachliche Analyse vor, die sich mit Gorbatschows Innenpolitik, seiner auf Rüstungsreduktion zielenden Außen- und Sicherheitspolitik sowie Ursachen und Problemen seines Reformkurses befasste: Die rückständige Produktionsweise in der Sowjetunion habe die Einführung von Entlohnung nach Gewinn notwendig gemacht, was freilich zu weiteren Ungerechtigkeiten und Arbeitslosigkeit führen werde. Die Menschen aber, die nicht länger unter Mangel leiden wollten, „werden die geforderte Anstrengung nicht mit einem angemessenen Konsum kompensieren können, deshalb sind sie eher skeptisch als begeistert". Wie Kelly nahm sie an, dass Gorbatschows innenpolitischer Erfolg „auf den außenpolitischen Erfolg angewiesen sein“ werde. Überdies teilte sie deren Auffassung, dass Gorbatschows neuer Kurs „eine historische Chance mit weltweiten Auswirkungen“ biete, die genutzt werden müsse. Einige Bedenken Elisabeth Webers aufnehmend, mahnte sie: „Ganz falsch finde ich es, mit unseren westlichen Ideologemen die Veränderungen in der UdSSR abzukanzeln." Freilich fand sie die eigene Unruhe darüber berechtigt, „bloß nicht alle Fehler zu wiederholen, die wir schon hinter uns haben“ - ein bisschen sollte Gorbatschow schon von den Grünen lernen ${ }^{74}$ !

Die Diskussion in der Fraktion ergab, dass man der Sowjetunion mehrheitlich eine düstere Zukunft prophezeite. Sie zeigte aber auch, wie gering das Interesse an der Sowjetunion und Osteuropa letztlich war. So blieb es dem Ökosozialisten Thomas Ebermann vorbehalten zu fragen, ob die Grünen „die Zielsetzung der erhöhten Produktivität überhaupt gut finden“. Der „Realo“ Hubert Kleinert mahnte, die Reichweite der Reformen nicht zu überschätzen; andere wie Willi Hoss (ehemals KPD/DKP) oder Udo Knapp (einst Funktionär des Sozialistischen Deutschen Studentenbunds) bezweifelten letztlich die Reformfähigkeit des sowjetischen Systems. Die Sowjetunion, so Knapp, treibe unter anderem aufgrund des Nationalitätenproblems „,in eine Krise hinein“. Jene, die sich wie der „Realo“ Otto Schily zu einer „Mischung von Hoffnung und Skepsis“ bekannten oder wie Christa Vennegerts nur die Frage aufwarfen, ob Gorbatschow seine Reformpolitik durchhalte, waren in der Minderheit. Schließlich zeigt sich der Mangel an Interesse daran, dass die Mehrheit es ablehnte, sich mit dem Entwurf eines Briefs an Gorbatschow zu befassen, der an die Fraktionsmitglieder verteilt worden war. Der

\footnotetext{
73 AGG, B.II.1, Nr. 1500, Helmut Lippelt, Thesen zur Einschätzung der „Gorbatschow-Politik“, 16.6.1987.

${ }^{74}$ Ebenda, Waltraud Schoppe, Zur Gorbatschow-Debatte, o.D.
} 
Vorschlag Schilys, die Diskussion darüber auf den folgenden Tag zu verschieben, wurde zwar akzeptiert; laut Protokoll der Sitzung am 17. Juni, die der Deutschlandpolitik gewidmet war, fand eine solche Diskussion allerdings nicht statt ${ }^{75}$.

Anders als die Mehrheit der Fraktionsmitglieder hatte Elisabeth Weber am 16. Juni gefordert: „Die Grünen sollten klar sagen, daß die Prozesse in der SU zu begrüßen sind.“ Überdies sei es sehr wichtig für die Grünen, „in der Ostpolitik einen Schwerpunkt zu sehen"76. Mit beiden Forderungen hatte sie bei Kelly, Lippelt und Schoppe Gehör gefunden, sich in der Fraktion aber nicht durchsetzen können. Jedoch gab sie nicht auf, sondern legte nach der Fraktionssitzung in der Kommune ihren Standpunkt nochmals ausführlich dar. Sie warb tendenziell dafür, Gorbatschows Revolution von oben und seinen Initiativen für mehr wirtschaftliche Effizienz größeres Verständnis entgegenzubringen. Sie stellte zwar explizit fest: „Gorbatschow ist kein Grüner“, mahnte aber, seine Vorstellungen über die Modernisierung der Sowjetunion sorgfältig zu prüfen und sich an dieser Debatte zu beteiligen. Kritik übte sie lediglich an den Wirtschaftsabkommen zwischen der Bundesrepublik und der Sowjetunion, unter anderem zur friedlichen Nutzung der Kernenergie. Hier zeichnete sich für sie „die Tendenz ab, dass Technikfetischisten aus Ost und West sich zusammenschließen, um gemeinsam ihr Atomprogramm zu betreiben“. Außerdem verdeutlichte sie, dass die Grünen „kein Interesse daran [hätten], daß westdeutsches Kapital sich günstige Produktionsbedingungen sucht in einem Land mit niedrigen Löhnen und ohne Streikrecht [...] und daß durch die Zusammenarbeit der Industriemanager das Nachdenken in beiden Gesellschaften über die ökologischen Folgen dieses Industriemodells verhindert wird“. Wie nicht anders zu erwarten, plädierte sie erneut dafür, mit den unabhängigen demokratischen Oppositionellen in Osteuropa zu kooperieren - diese blieben für viele grüne Konzepte „nach wie vor die einzigen Verbündeten"77.

Die Gegenmeinung wurde in derselben Zeitschrift von Udo Knapp vertreten, dem die Faszination, die Gorbatschow auslöste, eindeutig zu weit ging. Er äußerte sogar deutliche Kritik am Ehepaar Gorbatschow; dessen „sich im westlichen Luxus selbst darstellende großbürgerliche Attitüde [...] auf dem Hintergrund der Briefe von Menschen aus der Sowjetunion, die nicht genug zum Fressen haben“, machte ihn wütend. Entscheidend war für Knapp aber, dass Gorbatschows einzig erkennbare ökonomische Zielvorstellung „sich an westlichen kapitalistischen Entwicklungsmodellen mit all ihren ökologischen Verbrechen und sozialen Unge-

75 AGG, B.II.1, Nr. 569, Protokoll der Fraktionssitzung vom 16.6. 1987; AGG, B.II.1, Nr. 2136, Protokoll der Fraktionssitzung vom 17.6.1987. Es handelte sich um einen Briefentwurf von Gertrud Schilling und Petra Kelly, in dem um die Freilassung von Mathias Rust gebeten wurde, der mit einer Cessna am 31.5. 1987 auf dem Roten Platz gelandet und anschließend inhaftiert worden war. Der Briefentwurf wurde auf der Fraktionssitzung vom 23.6.1987 von Gertrud Schilling zurückgezogen. Diesen Hinweis verdanke ich Paul Kraatz.

76 AGG, B.II.1, Nr. 569, Protokoll der Fraktionssitzung vom 16.6.1987.

77 Elisabeth Weber, Zum Verhältnis der Grünen zu Gorbatschows Reformpolitik, in: Kommune 5 (1987), H. 8, S. 42-45, hier S. 43 f. 
rechtigkeiten orientiert" ${ }^{\text {"78 }}$. Beide Beiträge ähnelten zwar einander in der für die Grünen typischen Industriefeindlichkeit; jedoch brachte Weber aufgrund ihrer intimen Kenntnis der Situation in Osteuropa sehr viel mehr Verständnis für Gorbatschows wirtschaftspolitischen Reformkurs auf als Knapp.

\section{Neue Gesprächspartner und neue Themen 1987/88}

1987 und 1988 zeigte sich bei den Kontakten der Grünen in die Sowjetunion ein neuer Schwerpunkt. Hatten 1986 noch die Beziehungen zur politischen Führung überwogen - die Vertrauensgruppe wurde letztlich nur besucht, wenn GrünenDelegationen zu offiziellen Gesprächen in der Sowjetunion weilten -, intensivierte man nun die Verbindungen zu den sogenannten Informellen in der Sowjetunion. Nach wie vor pflegten jedoch nur wenige Personen diese Kontakte. Zwei Begegnungen grüner Politiker 1987 und 1988 mit Gorbatschow und Schewardnadse verdeckten jedoch, dass der Stern der Grünen in Moskau bereits im Sinken begriffen war, da die sowjetische Führung ihre Beziehungen zur Bundesregierung vertiefte.

Als Indiz für diese Entwicklung kann die Tatsache gelten, dass nach 1986 Einladungen zu einer offiziellen Visite in die Sowjetunion ausblieben. Als Anfang November 1987 die Bundesvorstandsmitglieder Jutta Ditfurth und Jürgen Maier Moskau besuchten, um an den Feierlichkeiten zum 70. Jahrestag der Oktoberrevolution teilzunehmen, bildeten sie nur eine von 178 Delegationen aus 123 Ländern. Gleichwohl nahm Gorbatschow die beiden mit der Begrüßung: „Ökologie und Sozialismus passen gut zueinander“, und Lob für Ditfurths Rede im internationalen Forum am 5. November ${ }^{79}$ direkt für sich ein. Auch der abschließende Bericht, in dem etwa die Bevorzugung der Grünen-Delegation bei den Wortmeldungen auf dem internationalen Forum hervorgehoben wurde, zeigt, wie lebendig die Illusion der eigenen Bedeutung für Gorbatschow noch war ${ }^{80}$. In ihrer Rede hatte Ditfurth die Oktoberrevolution als „eines der bedeutendsten Ereignisse der Geschichte" gepriesen, die amerikanische und bundesdeutsche Rüstungs- und Atompolitik kritisiert und generell vor der Nutzung von Atomenergie gewarnt ${ }^{81}$.

Elisabeth Weber war entsetzt über diese „kumpelhafte Anbiederung“: In Ditfurths Rede gebe es „kein Wort zum Pazifismus, zur Gewaltfreiheit, zur Kriegsdienstverweigerung, kein Wort zum Militarismus der Sowjetunion, kein Wort gegen deren autoritäres Staatsverständnis, kein Wort auch zu unserer Vorstellung von der Zukunft Europas, kein Wort zur demokratischen Opposition in Osteuropa“. Außerdem fehle jede „Spur der Betroffenheit über die Verbrechen der So-

\footnotetext{
${ }^{78}$ Udo Knapp, Zur Faszination der Rolle des „Großen Mannes“, in: Kommune 5 (1987), H. 8, S. $44 \mathrm{f}$.

${ }^{79}$ Dabei handelte es sich um eine zweitägige Großveranstaltung im Rahmen der Feierlichkeiten, auf der die Delegationen aus dem Ausland kurze Ansprachen hielten.

${ }^{80}$ AGG, PKA, Nr. 2795, Grünen-Pressedienst, Nr. 188/87, Bericht vom 12.11.1987 über die Reise der Delegation des Bundesvorstands nach Moskau vom 1.-8. 11. 1987.

${ }^{81}$ Ebenda, Rede Ditfurths vor dem Internationalen Forum im Kreml am 5. 11. 1987.
} 
wjetmacht“. Auf diese Weise vermittele sie ein falsches Bild von den Grünen: Denn es zeige „die Verführbarkeit der Grünen durch die Macht und dazu noch durch eine, die 70 Jahre lang mit brutalster Autorität herrschte“82. Diese Kontroverse verweist darauf, dass es bei den „Fundis“ auch Personen mit einer ausgesprochenen Sympathie für den Kommunismus sowjetischer Prägung gab, was andere, die sich den Oppositionellen im Ostblock verbunden fühlten, nicht nachvollziehen konnten. Möglicherweise lag Webers harsche Kritik daran, dass sie selbst von Gorbatschows Rede zum 70. Jahrestag der Oktoberrevolution enttäuscht war, zum einen wegen des mangelnden Muts zur historischen Wahrheit, zum anderen aber auch, weil darin „das gesamte Konzept der Industrialisierung weiter gerechtfertigt" worden $\mathrm{sei}^{83}$.

Gegen Ende seines dreitägigen Besuchs in Bonn vom 17. bis zum 19. Januar fand Eduard Schewardnadse noch Zeit für ein Gespräch mit Jutta Ditfurth und Petra Kelly. Während Ditfurth vor allem in Fragen der Abrüstung Einigkeit mit der Sowjetunion beschwor und nur vorsichtig nach der sowjetischen Einschätzung der Lage in Afghanistan fragte, war Kelly kritischer. Sie sprach sich für ein „blockfreies Europa“ aus und forderte damit den Abzug aller fremden Truppen, also auch der sowjetischen aus der ČSSR. Überdies mahnte sie erneut die Zusammengehörigkeit von innerem und äußerem Frieden an und kritisierte die Repressalien gegen Menschenrechtsgruppen in der Sowjetunion, die sie am eigenen Leib erfahren hatte, als Gert Bastian und ihr im vorangegangenen Dezember die Einreise zu einem Menschenrechtsseminar des Presseklubs „Glasnost“ verweigert worden war $^{84}$.

Trotz dieser Hindernisse wurden die Beziehungen zu den „Informellen“ in der Sowjetunion für die Grünen ab 1987 immer wichtiger, was auch damit zusammenhing, dass diese nun mehr Bewegungsfreiheit erhielten. Die Anzahl der Gruppen erhöhte sich insbesondere ab 1988 schlagartig; die Sowjetunion erlebte einen „Aufbruch [...] in den politischen Meinungspluralismus“ ${ }^{\text {“5 }}$. Die Grünen nutzten diese erweiterten Möglichkeiten. So nahm etwa der Mitarbeiter der Grünen-Fraktion Milan Horáček im Mai 1987 an einem Dialogtreffen von 300 Delegierten von

${ }^{82}$ AGG, PKA, Nr. 2536, Elisabeth Weber, Auf dem roten Teppich ausgerutscht? (15. 1. 1988), in: osteuropa-forum Nr. 19, März 1988, S. 7.

${ }^{83}$ AGG, B.II.3, Nr. 1078, Weber an Schily, 10.11.1987, Betr.: Moskau-Reise. Weitere Details über diese Moskau-Reise Schilys sind noch unbekannt.

${ }^{84}$ AGG, PKA, Nr. 470, Bericht vom Gespräch Grüne-Schewardnadse am 19.1.1988 (vgl. dazu auch Anm. 32). Zum Besuch Schewardnadses vgl. Claus Gennrich, Der Anschein des Nachgebens, in: Frankfurter Allgemeine Zeitung vom 20.1.1988. Zur geplanten Moskau-Reise Kellys und Bastians und der Visumsverweigerung vgl. AGG, B.II.3, Frieder [Wolf] an Kelly, 13. 11. 1987, Gesprächsnotizen mehrerer Gespräche mit Joanne Landy, betr.: Glasnost-Presseklub Menschenrechtsseminar; AGG, PKA, Nr. 469, Pressemitteilung Nr. 1136/87, Petra K. Kelly und Gert Bastian Visa für Moskau verweigert, 9. 12. 1987.

${ }^{85}$ Von Saal, KSZE-Prozess und Perestroika, S. 209-252, das Zitat S. 209. Bereits im Juli 1987 hatte der den Grünen nahestehende Osteuropa-Historiker Eichwede im Hinblick auf diese Vorgänge geschrieben: „In jedem Fall ist die heutige Konstellation ohne Vorbild in der Sowjetunion.“AGG, B.II.3, Nr. 1173, Wolfgang Eichwede, Moskau 1987: Wandel im Umgang mit den Bürgerrechten (Manuskriptabschluss: 5. 7. 1987). 
Friedensorganisationen aus 40 Ländern teil. Im November 1988 unternahmen Elisabeth Weber und Susanne Nies eine Reise nach Moskau, in der sie sich fast ausschließlich um Kontakte zu den „informellen Gruppen“ kümmerten. An ihren Berichten wird deutlich, dass die Grünen, die die Verhältnisse in der Sowjetunion selbst kennenlernten und Basiskontakte pflegten, größeres Verständnis für die Bedürfnisse der Menschen aufbrachten und auch den Wunsch nach mehr Konsumgütern eher nachvollziehen konnten. Elisabeth Weber reflektierte sogar im Anschluss an ihre Reise über das eigene Unvermögen, angemessen auf die Herausforderungen in der Sowjetunion zu reagieren. Die bundesdeutsche Gesellschaft kam ihr auf einmal, im Unterschied zur sowjetischen, „unnatürlich homogen vor“. Überdies erschien ihr die Bewertung bestimmter Vorgänge in der Bundesrepublik nach ihren Moskauer Erfahrungen als übertrieben: „Die Gedenkstunde im Bundestag, Jenningers Rede, die berechtigte Kritik daran, der Rücktritt, alles zusammen kam mir vor wie eine deutsche Hausfrau, die zwanghaft ihre Wäsche immer weißer zu waschen versucht, ohne zu sehen, daß nebenan die Leute nichts anzuziehen haben. Der satte Reichtum unseres Landes, materiell wie geistig, war mir kaum erträglich. " ${ }^{86}$ Eine solche Selbstkritik war jedoch bei den Grünen alles andere als selbstverständlich.

Es verwundert nicht, dass Weber für einen Ausbau der Beziehungen zu den „informellen Gruppen“ plädierte; diese hätten „große gesellschaftliche Bedeutung für die Selbsterziehung einer Gesellschaft, die kaum Erfahrungen mit demokratischer politischer Kultur hat“. Dabei sollten die Grünen zwar „keinerlei ,Bruderpartei'-Beziehungen und keinerlei Exklusivität herstellen“. Gleichwohl sprach sie sich „entschieden für eine Kooperation mit ,Memorial“ aus“, einer Gruppe, die „mit der Aufarbeitung des Stalinismus das [...] entscheidende Thema zur Herstellung von Demokratiefähigkeit der sowjetischen Gesellschaft behandelt und dies in einer Weise tut, die demokratisch ist, d.h. Menschen und ihre Erfahrungen zu Wort kommen läßt"87. Eine solche Kooperation zwischen „Memorial“" und der parteinahen Heinrich-Böll-Stiftung kam nicht zuletzt dank der Initiativen und Mahnungen Webers auch zustande. Die Böll-Stiftung gründete im September 1989 sogar einen „Arbeitskreis ,Memorial““, dem nicht nur Grüne angehörten, so dass der Kontakt institutionalisiert werden konnte ${ }^{88}$. Die Ablehnung des Stalinismus war offensichtlich bei den Grünen weit verbreitet, so dass eine Reihe grüner Abgeordneter 1988 auch den Appell „Für die vollständige Rehabilitierung der Opfer der Moskauer Prozesse“ unterschrieb. Die Hauptverwal-

\footnotetext{
${ }^{86}$ AGG, PKA, Nr. 469, Milan Horáček, Dialogtreffen von Friedensorganisationen in Moskau, 13.-15.5.1987, 12. 6. 1987; AGG, PKA, Nr. 470, Elisabeth Weber, Notizen über Gespräche mit einigen Mitgliedern „informeller Gruppen“ in Moskau im November 1988, 20.11.1988. Sie spielte darin auf die verunglückte Rede von Bundestagspräsident Philipp Jenninger zum 50. Jahrestag der Pogrome vom 9.11.1938 und dessen darauf folgenden Rücktritt an.

87 Ebenda.

${ }^{88}$ Zur Zusammensetzung des Arbeitskreises vgl. Memorial. Aufklärung der Geschichte und Gestaltung der Zukunft, hrsg. von der Heinrich-Böll-Stiftung, Bad Honnef 1989, S. 47 f. Diesen Hinweis verdanke ich Dr. Jürgen Zarusky. Vgl. dazu insgesamt AGG, B.II.3, Nr. 1041.
} 
tung A des Ministeriums für Staatssicherheit der DDR interpretierte dies als forcierte Aktivität „antikommunistische[r] Kreise der Grünen“89.

1988 kam zu den Reisezielen der Grünen in der Sowjetunion das Baltikum hinzu. So besuchten Elisabeth Weber und Ralf Fücks (ehemals Kommunistischer Bund Westdeutschland) von den Bremer Grünen Riga, wo sich am 3. September rund 100.000 Menschen an einer großen Aktion gegen das Ostseesterben beteiligten. Hier machten die Grünen „verblüffende Erfahrungen, die [ihre] Vorstellungen gründlich durcheinanderbrachten "90. Denn dass sich Proteste gegen Umweltverschmutzung auch mit nationalen Motiven und Forderungen nach kultureller wie politischer Autonomie verbinden konnten, war für sie völlig neu. Dennoch plädierte Weber im Anschluss an die Reise für eine Unterstützung dieser Forderungen, war sich aber sicher, dass die „Frage der Loslösung der baltischen Staaten von der UdSSR" nicht aktuell sei, so dass die Grünen dazu nicht Stellung beziehen müssten ${ }^{91}$. Andere, die von einem vierzehntägigen Bildungsurlaub im Baltikum berichteten, waren nicht so optimistisch, sondern sahen „die Gefahr eines sich verselbstständigenden Nationalismus, der Unabhängigkeit um jeden Preis zum Ziel hat" ${ }^{\text {"92 }}$.

Der Reformprozess in der Sowjetunion fand in den Führungszirkeln der Grünen allem Anschein nach weniger Aufmerksamkeit als noch ein Jahr zuvor, obwohl mit der Unionsparteikonferenz der KPdSU vom 28. Juni bis zum 1. Juli 1988 eine wichtige Etappe genommen und das Fundament für die ersten halbfreien Wahlen in der Sowjetunion gelegt wurde, die im März 1989 stattfinden sollten. Die Grünen beschränkten sich darauf, in einer Pressemitteilung die Ergebnisse der Konferenz zu begrüßen, von denen sie „die geforderte Abschaffung des Meinungsmonopols der Partei sowie die angestrebte Befristung der Tätigkeit von Parteifunktionären“ für besonders bedeutsam hielten ${ }^{93}$. Am 10. November 1988 meldete sich überdies Schily in der Bundestagsdebatte zum Besuch Helmut Kohls bei Gorbatschow zu Wort, warnte angesichts der verbesserten deutsch-sowjetischen Beziehungen vor „Wiedervereinigungsillusionen“ des Kanzlers und hoffte mit Bundespräsident Richard von Weizsäcker auf eine „systemöffnende Kooperation“, etwa bei der Umweltpolitik und bei der Umsetzung der „Vision eines gemeinsamen Europäischen Hauses“. Aus den sowjetischen Reformen resultiere

${ }^{89}$ Der Bundesbeauftragte für die Unterlagen des Staatssicherheitsdienstes der ehemaligen Deutschen Demokratischen Republik, MfS, HVA Nr. 52, Information über die Haltung der Grünen zu außen- und abrüstungspolitischen Fragen sowie zu ihrem Verhältnis zur DDR, 16.5. 1988, Bl. 183-187, hier Bl. 186.

${ }^{90}$ Ralf Fücks, Ökologie und Nationalismus. Lettische Irritationen - Erfahrungen einer Reise, in: Kommune 6 (1988), H. 11, S. 44-46, hier S. 44.

91 AGG, PKA, Nr. 470, Elisabeth Weber, Bericht vom 11.9.1988 an den AFI über eine Reise nach Riga vom 1.-6.9.1988.

${ }^{92}$ AGG, B.II.3, Nr. 1074, Wevers, Laing, Schneidewind, Protokoll des 14-tägigen Bildungsurlaubs: „Sowjetisches Baltikum: Avantgarde der Nation?“ (2.10.-16.10.1988). Das Zitat entstammt dem mit „Perestroika, Glasnost und der baltische Nationalismus“ überschriebenen Abschnitt.

93 AGG, PKA, Nr. 469, Pressemitteilung Nr. 635/86, Erklärung von Christa Vennegerts, 3.7. 1988. 
„eine neue historische Dimension für die europäische Zukunft“, blinde „Euphorie" sei aber nicht angebracht. Schily begrüßte die Chancen, die sich aus den sowjetischen Reformen für West- und Osteuropa ergeben konnten; zugleich sprach er sich ebenso gegen eine Abschottung des Westens in einer „Festung Westeuropa“ aus wie gegen die starre Erhaltung des Ostblocks durch die Sowjetunion. Für ihn war entscheidend, dass die „künftige europäische Architektur [...] sich nach den Interessen der europäischen Völker“ richtete, deren Wille „sich aber nur in einer europaweiten pluralistischen Demokratie verwirklichen“ lasse ${ }^{94}$. Damit hatte Schily eindeutig westliche Maßstäbe an die Reformen und Reformergebnisse angelegt; ein Dritter Weg, wie ihn offensichtlich viele andere Grüne favorisierten, kam für ihn als überzeugten „Realo“ nicht in Frage.

\section{Ein Dritter Weg? Perzeptionen und Diskussionen 1989}

Die spektakuläre Ankündigung Gorbatschows vor der Vollversammlung der Vereinten Nationen am 7. Dezember 1988, die sowjetischen Streitkräfte einseitig um 500.000 Mann und 10.000 Panzer zu reduzieren, die beginnenden Unruhen in Osteuropa und der Gorbatschow-Besuch in Bonn ließen die Debatten über Politik und Person des Generalsekretärs bei den Grünen erneut aufflammen. Für eine am 9. Mai 1989 geplante Fraktionssitzung arbeitete die BAG Frieden ein Papier aus, deren Verfasser vor allem im INF-Vertrag, in der jüngsten Abrüstungsinitiative Gorbatschows sowie in weiteren solchen Schritten, die dieser angekündigt hatte, eine „deutliche Bedrohungsminderung“ sahen. Die Sowjetunion betreibe zwar weiterhin Weltmachtpolitik und bewege sich nach wie vor „im Rahmen des Abschreckungssystems“; gleichwohl müssten jetzt einseitige Abrüstungsschritte des Westens folgen. Die Friedensbewegung und ihr verbundene Parteien hätten dabei „eine besondere Verantwortung [...], durch öffentlichen Druck die Entscheidungsträger der NATO-Staaten zur Beteiligung an dem von der UdSSR begonnenen Abrüstungsprozess zu veranlassen“, um so eine „Abrüstungsdynamik durch[zu]setzen“95. Die Grünen beharrten also weiter auf einseitigen Maßnahmen, ohne zur Kenntnis zu nehmen, dass alle Beteiligten vor allem auf bi- oder multilaterale Abrüstungsverhandlungen setzten.

Elisabeth Weber konstatierte im Frühjahr 1989 in einer umfassenden Analyse, dass in Osteuropa eine tiefgehende Systemkrise „offen ausgebrochen“ sei. Jetzt komme die Bewegung „,on unten“ in Gestalt der „,informellen Gruppen und im Gewand nationaler Konflikte im sowjetischen Vielvölkerstaat; überdies seien die Strukturprobleme der Wirtschaft unberechenbar. Die damit verbundenen Auseinandersetzungen drohten „alle Reformansätze zunichte zu machen“. Die Grünen

\footnotetext{
${ }^{94}$ Deutscher Bundestag, Stenographischer Bericht, 11. Wahlperiode, 106. Sitzung am 10.11. 1988, S. $7292 \mathrm{f}$.

${ }^{95}$ AGG, B.II.1, Nr. 2107, BAG Frieden, Abrüstungsdynamik durchsetzen - Wir machen den zweiten und dritten Schritt. Die friedenspolitische Antwort der Grünen auf Gorbatschow, Mai 1989. Als Verfasser der Vorlage wurde in der Fraktionssitzung am 23.5. 1989 der Bundestagsabgeordnete Tay Eich genannt; vgl. AGG, B.II.1, Nr. 2800.
} 
müssten daher einsehen, dass es nicht mit der Zustimmung zu Gorbatschows Abrüstungsinitiativen getan sei. Osteuropa stehe „vor gigantischen innenpolitischen Aufgaben“, bei denen der Westen nicht viel tun könne: Das „Schicksal von Gorbatschow wird sich nicht an der Reaktion des Westens, sondern am innenpolitischen Kräfteverhältnis entscheiden“. Sie ließ auch keinen Zweifel daran, dass im „Systemkonflikt zwischen Ost und West [...] plötzlich der Westen offen als Sieger“ dastehe und die Blockstruktur in Bewegung gerate, was neue Möglichkeiten schaffe. Einen Dritten Weg zwischen Ost und West, mit dem viele Grüne liebäugelten, hielt sie dabei jedoch für ausgeschlossen. Doch so klar ihre Analyse auch war, so wenig konkret waren ihre Hinweise auf die Schlussfolgerungen, die die Grünen daraus ziehen sollten. Als Oppositionspartei könnten die Grünen „nicht viel mehr machen, als einerseits bei der Herausbildung neuer gesellschaftlicher Milieus helfen und andererseits einige ostpolitische Akzente setzen“. Diese wurden zwar näher ausgeführt, liefen aber letztlich auf die alten Themen und Rezepte hinaus - Ökologie, Kooperation mit der demokratischen Opposition, Plädoyer für „eine gesamteuropäische Orientierung “96.

Wenngleich im Frühjahr 1989 die revolutionären Entwicklungen in Mittel- und Osteuropa noch nicht absehbar waren, entging aufmerksamen Beobachtern im Westen die bis dahin ungeahnte Dynamik der Vorgänge nicht. Umso erstaunlicher ist es, dass die Bundestagsfraktion der Grünen dieser Frage kaum Aufmerksamkeit entgegenbrachte. Nachdem das Thema in der Sitzung am 9. Mai „aufgrund der bereits fortgeschrittenen Zeit“, den „Ermüdungserscheinungen der Fraktion“97 und „mangels Teilnahme der MdBs“98 vertagt worden war, kam es am 23. Mai wieder auf die Agenda. Dabei machte der Abgeordnete Tay Eich von den „Fundis“, der das Papier der BAG Frieden verfasst hatte, eingangs darauf aufmerksam, dass auch diesmal zu wenige Bundestagsabgeordnete anwesend seien, „um einen Beschluß zu erwirken“. Es kam zwar zu einer Aussprache; der Vorschlag, ein Meinungsbild festzuhalten, wurde jedoch mit fünf zu vier Stimmen abgelehnt.

Wenig später gerieten Gorbatschow und seine Reformen erneut in den Fokus der Grünen, als der Generalsekretär vom 13. bis zum 15. Juni 1989 die Bundesrepublik besuchte. Ursprünglich war vorgesehen, dass Gorbatschow auch Vertreter der Grünen empfangen und bei dieser Gelegenheit einen Brief des Bundesvorstands und der Fraktion entgegennehmen sollte. Das Schreiben wurde Gorbatschow zwar am 31. Mai über die sowjetische Botschaft zugeleitet und erschien am 15. Juni in der Frankfurter Rundschau $u^{99}$. Das Treffen wurde aber gestrichen, und die Grünen mussten sich mit einem Gespräch mit Gorbatschows Berater Alexan-

${ }^{96}$ AGG, PKA, Nr. 470, Elisabeth Weber, Grüne Ost-Politik - gibt es das?, März/April 1989, mit handschriftlichem Vermerk: „Vorlage zur Diskussion in der Ost-West-AG“.

${ }^{97}$ AGG, B.II.1, Nr. 2800, 82. Fraktionssitzung am 9.5.1989.

98 Ebenda, 83. Fraktionssitzung am 23.5. 1989. Dieses und alle weiteren Zitate entstammen diesem Protokoll.

${ }^{99}$ AGG, B.II.1, Nr. 2147, 84. Fraktionssitzung am 30.5. 1989; AGG, PKA, Nr. 471, Bundesvorstand und Fraktionsvorstand an Gorbatschow, 31.5. 1989. Zum Inhalt vgl. den Anfang dieses Aufsatzes. 
der Jakowlew und anderen sowjetischen Delegationsmitgliedern begnügen ${ }^{100}$ ein weiterer Hinweis auf den Bedeutungsverlust der Grünen für die sowjetische Führung, die nun vor allem die Beziehungen zur Bundesregierung pflegte.

Die Grünen nutzten den Besuch vor allem zu Kritik an Bundeskanzler Kohl: „,Perestroika‘ kann nicht heißen, in Osteuropa soll sich alles ändern, und in der Bundesrepublik bleibt alles beim alten." Die Bundesregierung solle den an Gorbatschow gepriesenen Mut zur Erneuerung endlich selbst aufbringen und „einen seit Jahren überfälligen eigenen Beitrag der Bundesrepublik zum Abrüstungsprozeß in Europa“ liefern ${ }^{101}$. Daran knüpfte Helmut Lippelt im Bundestag an, als er Kohl vorwarf, sich „hinter dem Raketenzaun der NATO“ zu verschanzen, während Gorbatschow von der Perestroika spreche, die „auch ein Bestandteil zunehmender Demokratisierung der Weltordnung"sei. Überdies bemängelte er, dass in der von Gorbatschow und Kohl unterschriebenen „Gemeinsamen Erklärung viele schöne Prinzipien“, wie das Überleben der Menschheit, die Rettung der Umwelt und die Überwindung von Hunger und Armut, enthalten seien; aus den abgeschlossenen Abkommen gehe jedoch eine Überbetonung der wirtschaftlichen Beziehungen hervor. Es komme letztlich nicht darauf an, ob Kapitalismus oder Sozialismus das bessere System seien, sondern es gehe darum, „wie sich beide bei den neuen Aufgaben des Umweltschutzes bewähren“. Erneut mahnte er an, „daß die Veränderungen in Osteuropa auch Anstöße zu Veränderungen bei uns bedeuten müssen“. Damit instrumentalisierte Lippelt Gorbatschows Reformkurs, um die Bundesregierung als Bremser im Abrüstungsprozess zu brandmarken. Die weit verbreitete „Gorbimanie“ interpretierte er als „Wunsch nach einer besseren Zukunft, nach Überwindung der alten Feindbilder, nach Überwindung der Militärblöcke, der gegenseitigen Bedrohung, der Überrüstung, der Abgrenzung und der geschlossenen Grenzen“. So projizierte er grüne Wunschvorstellungen in die Begeisterung der Menschen ${ }^{102}$. Deutlich wird, dass die Grünen Gorbatschow weiterhin an ihren eigenen Idealen maßen und bei aller Sympathie für seine Abrüstungsinitiativen gegenüber seiner Wirtschaftspolitik skeptisch blieben.

Während die Bundestagsfraktion der Grünen über Gorbatschow und seine Politik kaum mehr diskutierte, gewann die Idee eines „Perestroika“-Kongresses Gestalt, die aus dem sogenannten Linken Forum stammte. Im April 1989 waren die Pläne bereits so konkret, dass Jürgen Reents, ein Protagonist dieser Gruppierung, unter anderem den Bundesvorstand und den Fraktionsvorstand der Grünen über das Vorhaben informieren konnte. Ziel des Kongresses war es, „eine umfassende Diskussion über die Perestroika in der Sowjetunion zu führen und Überlegungen daraus für die gesellschaftspolitische Debatte in der Bundesrepublik zu gewin-

${ }^{100}$ AGG, B.II.1, Nr. 2107, Pressemitteilung Nr. 514/89, Grüne sprachen mit Alexander Jakowlew, 15.6.1989.

101 AGG, PKA, Nr. 1588, Pressemitteilung Nr. 502/89, Gorbatschow-Besuch und „Gemeinsame Erklärung“, 13.6.1989.

102 Deutscher Bundestag, Stenographischer Bericht, 11. Wahlperiode, 150. Sitzung am 16.6.1989, S. 11197-11199. 
nen“. Die Konferenz sollte gemeinsam mit der West-Berliner Alternativen Liste organisiert werden und im Frühjahr 1990 mit Gästen aus der Sowjetunion in WestBerlin stattfinden. Damit war auch ein klares Signal verbunden: die „Abkehr von der ,Ost-West-Frontstadt“ zu einer ,Ost-West-Dialogstadt“" ${ }^{\prime 103}$. Reents zufolge stimmte der Bundesvorstand am 29. Mai „im groben“ zu, auch der Bundeshauptausschuss befürwortete das Projekt am 17. Juni 1989 im Großen und Ganzen. Am 12. September lag zwar immer noch kein überarbeitetes Konzept, aber dafür ein neuer Arbeitstitel vor: „Grenzüberschreitungen. Perestroika Ost - Alternativen West: Wege zum gemeinsamen europäischen Haus?"104 Dies zeigt schon, wie realitätsfern Reents und seine Mitstreiter waren, die trotz der unübersehbaren Krise des realsozialistischen Systems - zwei Tage zuvor hatte die ungarische Regierung die Grenze für Flüchtlinge aus der DDR geöffnet - noch an einen Dritten Weg zwischen Sozialismus und Kapitalismus glaubten, den sie gemeinsam mit der Sowjetunion beschreiten wollten.

Obwohl die Vorbereitungsgruppe für den Kongress 270.000 DM benötigte, unterstützte Ralf Fücks vom Bundesvorstand am 12. Dezember die Idee, die „durch die revolutionären Ereignisse in Ost-Mitteleuropa noch an politischer Aktualität gewonnen“ habe, empfahl aber den Untertitel „Auf der Suche nach dritten Wegen“. Gleichzeitig mahnte er an, die Veranstaltung für die Reformbewegungen aus Ostmitteleuropa zu öffnen und auch deren Protagonisten einzuladen ${ }^{105}$. Horáček hatte letzteres bereits zu einem früheren Zeitpunkt vorgeschlagen, war aber von Reents mit der Bemerkung abgewiesen worden, „die Prozesse in den übrigen Ländern seien dafür noch nicht reif genug“. Jetzt wiederholte er seine Forderung und gab zu bedenken, dass es seines Erachtens „nicht um die Suche nach einem 3. Weg [gehe], sondern um die Suche nach demokratischen europäischen Rechtsstaaten"106. Während das Linke Forum noch in alter Tradition vor allem mit den sowjetischen Machthabern ins Gespräch kommen wollte und dabei die wachsende Bedeutung der Bewegung von „unten“ ausblendete, waren diejenigen, die mit den Verhältnissen in den ostmitteleuropäischen Staaten vertraut waren und eigene Kontakte mit den dortigen Oppositionellen unterhielten, sehr viel eher zu einem zutreffenden Urteil in der Lage.

Die Organisatoren des Linken Forums ließen sich indes kaum von dieser Kritik beeindrucken. Zwar versuchten sie nun auch, sowjetische „Informelle“ einzubeziehen - womit sie auf den Unwillen ihrer offiziellen sowjetischen Partner stießen -, hielten aber zunächst an einem weitgehend bilateralen Konzept fest ${ }^{107}$. Schließlich teilte Fücks am 20. März 1990 dem Fraktionsvorstand, Lippelt und der OstWest-AG mit, der Kongress könne nicht wie geplant stattfinden, weil die Finanzierung nicht gesichert und „die politische Unterstützung innerhalb der Grünen

103 AGG, B.II.3, Nr. 1035, Reents an Bundesvorstand, Fraktionsvorstand u.a., 15.4. 1989: Projekt Perestroika-Kongress (mit Anlagen).

104 AGG, B.II.1, Nr. 2278, Reents an Lippelt, 28.9. 1989; Protokoll Vorbereitungsgruppe Perestroika-Kongress, 12.9.1989.

105 AGG, B.II.3, Nr. 1035, Fücks an Vorbereitungsgruppe Perestroika-Kongress, 12. 12. 1989.

106 AGG, B.II.1, Nr. 2278, Horáček an Fücks, 15. 12. 1989.

${ }^{107}$ AGG, B.II.3, Nr. 1035, Bundesweite Perestroika-Koordination, 28. 2. 1990. 
infragegestellt“ sei. Das Vorhaben sei „nicht zuletzt an der - vornehm ausgedrückt - distanzierten Haltung der Bundestagsfraktion und des Ost-West-AK [sic] gescheitert" ${ }^{108}$. Lippelt und Weber wiesen diese Vorwürfe empört zurück. Lippelt hatte sich sehr wohl in der Fraktion für den Kongress eingesetzt, bis er erfuhr, dass geplant war, sowohl den tschechoslowakischen Staatspräsidenten Vaclav Havel als auch den polnischen Arbeitsminister Jacek Kuroń einzuladen, die zwar äußerst prominent, aber nicht abkömmlich waren. „[N] ach solch durchsichtiger Hochstapelei“" war Lippelt die Lust vergangen, sich weiter zu engagieren ${ }^{109}$. Weber verwies darauf, dass die Vorbereitungsgruppe selbst eine ernsthafte Kooperation mit der Ost-West-AG abgelehnt habe, da deren Mitarbeit „nicht ins strömungspolitische Profil des geplanten Kongresses paßte“. Die Ost-West-AG sei überdies nach der Lektüre der Vorbereitungspapiere zu dem Ergebnis gekommen, dass die ganze Sache letztlich unseriös sei ${ }^{110}$.

Die Auseinandersetzung um diesen Kongress, der nie stattfand, war einerseits Teil der grünen Flügelkämpfe; andererseits steht er aber auch für divergierende Perzeptionen der Sowjetunion unter Gorbatschow, die aus zwei unterschiedlichen Zugangsweisen resultierten: Das Linke Forum glaubte 1988/89, mit einer reformierten, aber weiterhin sozialistischen Sowjetunion einen seit langem von Seiten der „undogmatischen Linken“ angestrebten Dritten Weg zwischen Ost und West beschreiten zu können, wobei man sich fast ausschließlich auf die sowjetische Führung konzentrierte. Lippelt, Weber und ihre Mitstreiter hingegen gingen weniger von eigenen Wunschvorstellungen als von den bekannten Realitäten aus. Die meisten Menschen in Osteuropa wollten keinen Dritten Weg, sondern den demokratischen Rechtsstaat. Die Frage, ob und wie die Sowjetunion unter diesen Bedingungen weiter überleben sollte, war für sie dabei letztlich zweitrangig.

\section{7. „Von der Realität überrollt“: Die Grünen und die Sowjetunion 1990}

Als infolge der friedlichen Revolution in der DDR die deutsche Wiedervereinigung ermöglicht wurde, bedeutete dies eine nicht nur von den Grünen völlig unerwartete Renaissance des Nationalstaats in Europa. Elisabeth Weber hatte zwar die Staaten Osteuropas für nicht stabil gehalten, aber nationales Denken kategorisch abgelehnt: „Ein politisches Denken in den Interessen des Nationalstaats“, so ihre Überzeugung, „gehört nicht in das Europa der Jahrtausendwende.“ Anfang des Jahres 1990 stellte sie „mit Bestürzung“ fest, wie sehr sie sich, „trotz aller blockübergreifenden Konzepte, auf den Status quo eingestellt hatte“. Die Grünen, so Weber weiter, seien „in ihrem Selbstverständnis von der deutsch-deutschen Entwicklung überrumpelt worden"111. Obwohl die Grünen sich im Frühjahr

\footnotetext{
108 Ebenda, Fücks an Fraktionsvorstand, Lippelt, Ost-West-AG, o. D. [20.3. 1990].

${ }^{109}$ Ebenda, Lippelt an Fücks, 21.3.1990.

${ }^{110}$ Ebenda, Weber an Fücks und Vorbereitungsgruppe Perestroika-Kongress, 22. 3. 1990.

${ }^{111}$ Elisabeth Weber, Bericht über meine ersten Erfahrungen der Zusammenarbeit von Grünen und Bündnis 90 [Dezember 1990], in: Grünes Gedächtnis (2009), S. 23-37, hier S. 28.
} 
1990 mit der deutschen Einheit arrangierten ${ }^{112}$, blieben sie äußerst skeptisch gegenüber der Politik der Bundesregierung, die ein wiedervereinigtes Deutschland in der NATO anstrebte.

Nachdem Gorbatschow am 31. Mai einer deutschen NATO-Mitgliedschaft gegenüber Präsident Bush verklausuliert und am 15./16. Juli in Moskau und Archys gegenüber Kohl endgültig zugestimmt hatte, bat Fraktionssprecher Willi Hoss den „Realo“ Wolfgang Bruckmann um eine Einschätzung der neuen Situation ${ }^{113}$. Bruckmanns Papier enthielt unter anderem heftige Kritik an der Haltung der Grünen. Diese hätten sich ,in den drei großen außen- und sicherheitspolitischen Wendepunkten der letzten Jahre als kaum handlungsfähig erwiesen“. Erstens hätten sie trotz „der Realität des INF-Vertrages“ weiter „die Kündigung des NATODoppelbeschlusses verlangt und damit dogmatisch an einseitiger Abrüstung festgehalten“. Sie hätten, zweitens, zu lange auf der Zweistaatlichkeit beharrt und es, drittens, abgelehnt, „,sich auf die absehbare NATO-Mitgliedschaft eines vereinten Deutschlands einzulassen“. Obwohl Bruckmann die Grünen schon am 10. Mai vor die Wahl gestellt hatte, entweder „ein zweites Mal von der Realität überrollt“ zu werden oder einen Zukunftsentwurf auf der Basis der deutschen NATO-Mitgliedschaft zu erarbeiten, habe die Fraktion kein einziges Mal über diese Fragen beraten. Einzelne Abgeordnete hätten lediglich Stellungnahmen „nach bekanntem Muster“ abgegeben, denen zufolge eine deutsche NATO-Mitgliedschaft Gorbatschow und seine Reformen gefährde; grundsätzlich habe es „viel Verständnis nur für die sowjetische Position“ und „antiwestliche Affekte“ gegeben ${ }^{114}$. Die grünen Abgeordneten, die sich überhaupt zu dem Thema äußerten, hatten aus traditioneller Abneigung gegen die amerikanische Regierungspolitik und gegen die NATO sowie aus einer grundsätzlichen Pro-Gorbatschow-Stimmung heraus die Augen vor der Realität verschlossen.

Doch wie sollten sich die Grünen nun positionieren? Elisabeth Weber schrieb dazu in der Kommune, die Grünen seien „wie gelähmt durch den Schock, den die Entwicklungen in Osteuropa für viele bedeuten“, und müssten ,jetzt dafür bezahlen, dass sie die seit vielen Jahren schwelende Krise in Osteuropa regelrecht verdrängten“. Sie hätten im Gegenteil eine Reihe von Tabus gepflegt, wie etwa die Ablehnung der nun von den Osteuropäern zusammen mit dem Rechtsstaat angestrebten Marktwirtschaft, und sie hätten vielfach „den Zusammenhang von Frieden und Menschenrechten auseinander[ge]rissen“. Drei Zukunftsszenarien hielt Weber für denkbar: ein düsteres, in dem in der Sowjetunion „eine Militärdiktatur oder ein Faschismus russischen Stils“ errichtet werde, Osteuropa zerfalle, EG-Europa sich als „schimmernde Burg“ organisiere, Deutschland vereinigt und „die Mauer [...] an der Oder-Neiße-Grenze neu errichtet" werde; ein konservativ-autoritäres mit stagnierender Entwicklung in der Sowjetunion aufgrund eines innenpolitischen Patts zwischen traditionellen und Reformkräften, einem Osteuropa, in dem sich „autoritär-nationalistische Muster“ durchsetzten, Deutschland zu

\footnotetext{
112 Vgl. Wick, Die Mauer muss weg, S. 272-280.

113 AGG, B.II.1, Nr. 2012, Hoss, Schoppe, Vollmer an Fraktionsvorstand und AFI, 30. 7. 1990.

114 Ebenda, Bruckmann an Vorstand, z. H. Hoss, 20. 7.1990.
} 
Kohls Bedingungen vereinigt werde und die EG mit den schwachen osteuropäischen Staaten kooperiere; schließlich ein demokratisches, in dem es kommunistischen Reformpolitikern in der Sowjetunion und in Osteuropa zusammen mit demokratisch-informellen Bewegungen gelänge, „einen Prozeß der demokratischen Umgestaltung von Wirtschaft und Gesellschaft einzuleiten“, DDR und Bundesrepublik „von unten“ zusammenwüchsen, aber zwei Staaten blieben, und Europa sich „auf der Basis des KSZE-Prozesses“ zusammenschlösse, gemeinsam ökologische Umbauschritte einleite, entmilitarisiert sei, kooperativ mit der Dritten Welt zusammenarbeite und ,in dem die Menschen [...] freundschaftlich und sich gegenseitig bereichernd zusammenleben"115. Das dritte Szenario zeigt, dass auch Weber von der Vision einer grün gefärbten europäischen Zukunft nicht lassen wollte, wenngleich sie skeptisch war, ob diese unter den damaligen Bedingungen verwirklicht werden konnte. Die Schlussfolgerungen aus ihren Überlegungen für die operative Politik blieben wieder sehr allgemein. Die Ost-West-AG war sich allerdings bei ihrem Treffen vom 15./16. Dezember 1990 einig, „daß eine Arbeitsgruppe zu Osteuropa im Spektrum Die Grünen/Bündnis 90 weiterexistieren" müsse. Deren Aufgaben sollten weiterhin vor allem Basiskontakte nach Osteuropa umfassen, um dort Partner „für eine ökologische, demokratische und soziale Politik“" zu suchen ${ }^{116}$.

\section{Fazit}

Die Beurteilung Gorbatschows und seiner Politik fiel den Grünen - wie auch den anderen politischen Parteien - zunächst nicht leicht, was auch an den widersprüchlichen Signalen lag, die die Bundesrepublik aus der Sowjetunion erreichten. Dennoch erhielt ihr Gorbatschow- und Sowjetunion-Bild ab 1986 erste, wenngleich zum Teil noch verschwommene Konturen. Die Außen- und Abrüstungspolitik des Generalsekretärs bestätigte weitgehend das grüne Weltbild, in dem die Sowjetunion zwar nicht als Freund, aber auch nicht als Feind galt. Die Grünen wollten nicht in den Chor jener einstimmen, die „Angst vor den Russen“ propagierten, um eine weitere Aufrüstung zu rechtfertigen. Als Gorbatschow mit Rüstungskontroll- und Abrüstungsinitiativen an die Öffentlichkeit trat, stand daher für die Grünen, anders als für weite Teile der Regierungsparteien und viele Sowjetexperten, deren Ernsthaftigkeit von Anfang an fest. Die Vereinigten Staaten unter Ronald Reagan und die NATO, denen die Grünen einen echten Abrüstungswillen absprachen, bestätigten dieses Weltbild, da sie auf die Vorschläge Gorbatschows zu zögerlich reagierten oder diese sogar torpedierten - wie etwa beim Gipfel in Reykjavik. Gleichwohl entsprachen die sowjetischen Initiativen nicht genau ihren Vorstellungen, weil Moskau am Bilateralismus festhielt, die Grünen aber fast ausschließlich auf einseitige Abrüstung setzten.

\footnotetext{
${ }^{115}$ Elisabeth Weber, Große Hoffnung, große Angst, in: Kommune 8 (1990), H. 2, S. $26-28$.

${ }^{116}$ AGG, B.II.3, Nr. 1205, Elisabeth Weber, Protokollnotiz einer Diskussion über die zukünftigen Aufgaben einer Ost-West-Arbeitsgruppe im Umkreis von Die Grünen/Bündnis 90, 21.1.1991.
} 
In der Beurteilung der Innenpolitik Gorbatschows taten sich die Grünen schwerer. 1985/86 wurde ihr Gorbatschow-Bild noch primär durch den sowjetischen Krieg in Afghanistan, fortgesetzte Menschenrechtsverletzungen und das unnachgiebige Vorgehen gegen Dissidenten beeinträchtigt. Als Sacharow Ende 1986 aus der Verbannung zurückkehren durfte und danach die politischen Gefangenen sukzessive freigelassen wurden, änderte sich auch die Vorstellung einer durch und durch repressiven Sowjetunion. Dagegen waren die Grünen 1986 über das sowjetische Verhalten angesichts der Reaktorkatastrophe von Tschernobyl vorübergehend sehr enttäuscht, auch wenn sie das nach außen kaum zu erkennen gaben: Gorbatschow war damit anscheinend auf die Seite der gegnerischen „Atomkoalition“ gewechselt. Dies trübte das Bild zwar bis Ende 1986, bevor sich die Grünen verstärkt anderen Themen zuwandten. Was sie fast durchgehend an Gorbatschow und seinen Reformversuchen störte, war deren wirtschaftspolitischer Schwerpunkt: Da sie die moderne Industriegesellschaft und die westliche Marktwirtschaft ablehnten, standen sie auch Gorbatschows Bemühungen auf dem Feld der Ökonomie kritisch gegenüber. Das betraf die Bemühungen um mehr wirtschaftliche Effizienz ebenso wie seine Initiative zu verstärkter Kooperation mit der westdeutschen Wirtschaft. Die meisten Grünen unterstellten dem Generalsekretär sogar, marktwirtschaftliche Verhältnisse etablieren zu wollen, obwohl sich Gorbatschow damit bis 1990 Zeit ließ. Hier zeigt sich, wie das grüne Feindbild die Realität überlagerte.

Zwar einte die Skepsis gegen die sowjetische Wirtschaftspolitik die verschiedenen Flügel der Grünen, gleichwohl lassen sie sich auch in ihrer Einschätzung Gorbatschows und der Sowjetunion nicht über einen Kamm scheren. Auf der einen Seite standen hier noch Personen, die wie Jutta Ditfurth unverhohlene Sympathien für das mit der Oktoberrevolution eingeleitete sozialistische Experiment Sowjetunion äußerten, oder Annemarie Borgmann, die in der Sowjetunion immerhin einen „Hoffnungsträger“ in puncto Abrüstung sah. Auf der anderen Seite standen jene, die wie Elisabeth Weber nicht müde wurden, die Schattenseiten der sowjetischen Diktatur zu benennen. Inwieweit Gorbatschow durch seine Persönlichkeit dieses Bild änderte, hing wesentlich vom Betrachter ab. Petra Kelly mit ihrer emotionalen Beurteilung Gorbatschows nahm hier vielleicht eine Sonderstellung ein; ansonsten scheint sich die „Gorbimanie“ unter den Grünen in Grenzen gehalten zu haben.

Allerdings wandelten sich die Urteile und Bewertungen seit 1987. Nun ging es nicht mehr nur um den Kurs Gorbatschows, sondern auch um die Frage, ob er sich damit auch durchsetzen könne. War er in der Lage, die Regale in den Geschäften zu füllen, um so die Konsumwünsche der Sowjetbürger zu befriedigen und damit den Rückhalt für seine Politik unter der Bevölkerung zu erhalten? Konnte er sich gegen seine Widersacher im Partei- und Staatsapparat sowie in den Streitkräften durchsetzen? Dass der Westen Gorbatschow dabei unterstützen konnte, indem er ihm bei der Abrüstung entgegenkam, war bis Ende 1988 eine weit verbreitete Meinung; erst im Frühjahr 1989 räumte Elisabeth Weber angesichts der Krise des realen Sozialismus mit diesem Irrglauben auf. Dass nicht nur die Wirtschaft, sondern auch die Nationalitätenfrage Gorbatschow vor ungeahnte 
Herausforderungen stellte, wurde den Grünen richtig erst ab 1988 bewusst. Sie machten dabei insbesondere im Baltikum die Erfahrung, dass das Engagement für Umweltschutz, Demokratie und nationale Eigenständigkeit eine für sie bis dahin unbekannte Verbindung eingehen konnten.

Das Gorbatschow-Bild und die transnationalen Beziehungen der Grünen in die Sowjetunion waren untrennbar miteinander verbunden. Die Grünen, die von sowjetischer Seite auch entsprechend hofiert wurden, betrachteten sich und die Friedensbewegung als politische Bündnispartner Gorbatschows. Auf ihr Zusammenspiel führten sie auch das INF-Abkommen vom Dezember 1987 zurück. Da die Sowjetunion die Grünen als Alliierte für ihre Friedenspolitik benötige, glaubten letztere auch, auf Gorbatschows Innenpolitik Einfluss nehmen zu können. Freilich ließ ihre Bedeutung für Gorbatschow seit 1987 objektiv nach, was sie zunächst nicht wahrnahmen. Erst 1988/89 muss ihnen dies deutlich geworden sein, etwa als sie von Gorbatschow anlässlich seines Bonn-Besuchs nicht empfangen wurden. Die Beziehungen zur sowjetischen Führung hatten folglich auch weitgehende Rückwirkungen auf die Selbstwahrnehmung der Grünen.

Die Basiskontakte nach Osteuropa und in die Sowjetunion wurden nur von einer kleinen Gruppe aufrechterhalten. Bei den Grünen, die sich hier engagierten, entwickelte sich jedoch eine größere Sensibilität gegenüber den Dissidenten sowie den dortigen Gesellschaften mit ihren Bedürfnissen und Wünschen. Elisabeth Weber und ihre Mitstreiter brachten sehr viel mehr Verständnis für die Konsumwünsche der Osteuropäer auf als Parteifreunde, die sich nur theoretisch mit dem Ostblock beschäftigten. Wichtiger war indes Webers Erkenntnis, dass es den Oppositionellen in der Sowjetunion und Osteuropa nicht um einen Dritten Weg, sondern um einen demokratischen Rechtsstaat ging. Aufgrund ihres Austauschs mit den dissidenten Gruppen war ihnen der Wert des demokratischen Rechtsstaats mit Gewaltenteilung und Verpflichtung zur Wahrung der Menschenrechte durchaus bewusst, so dass sie bei aller Kritik an den Verhältnissen in der Bundesrepublik den Unterschied zwischen Diktatur und Demokratie noch deutlich zu ziehen vermochten. Anders verhielt es sich mit einigen Exponenten des fundamentalistischen Flügels der Grünen, die noch 1989 mit dem immer stärker erodierenden sowjetischen Regime über einen Dritten Weg diskutieren wollten.

Am Ende wurden die Grünen gleich welcher Couleur „von der Realität überrollt". Wenngleich manche von ihnen die Ostblockstaaten als instabil eingeschätzt hatten, wurden sie von dem sowjetischen Rückzug aus Osteuropa und der Wiedervereinigung Deutschlands überrascht, denn sie hatten, wie so viele, die Beharrungskraft des Nationalstaats unterschätzt. Zahlreiche Grüne hatten sich zwar im Vereinigungsprozess noch auf die Seite Gorbatschows gestellt und sich auch gegen eine NATO-Mitgliedschaft des vereinigten Deutschland ausgesprochen. Als die Entwicklung jedoch einen anderen Verlauf nahm und die Grünen im Dezember 1990 eine verheerende Wahlniederlage verkraften mussten, verschwanden Osteuropa und die Sowjetunion fast völlig von ihrer Agenda. Noch einmal, beim Putsch gegen Gorbatschow vom 19. bis zum 21. August 1991, riefen sie zu Protestkundgebungen vor der sowjetischen Botschaft in Bonn und dazu auf, die Krise in der Sowjetunion „durch freie Wahlen, [und] nicht durch Panzer“ zu lösen. In der 
Niederlage der Putschisten sahen sie dann einen „großartige[n] Erfolg für Boris Jelzin und die Demokratiebewegung “117. Auch für die Grünen verblasste der Stern Gorbatschows, den sie nie so euphorisch wie andere in der Bundesrepublik umjubelt hatten.

117 AGG, B.II.3, Pressedienst Bündnis 90/Die Grünen, Nr. 208/91, Bestürzung über Ausschalten Gorbatschows / Für Demokratie in der Sowjetunion demonstrieren, 19. 8. 1991; ebenda, Nr. 215/91, Großartiger Erfolg für die Demokratiebewegung in der Sowjetunion/Jelena Bonner frei, 21.8.1991. 